\title{
Modulation of OSCP mitigates mitochondrial and synaptic deficits in a mouse model of Alzheimer's pathology
}

\author{
Esha Gauba $^{1 \#}$, Shaomei Sui ${ }^{1 \#}$, Jing Tian ${ }^{1}$, Christopher Driskill $^{2}$, Chunxiao Yu ${ }^{1}$, Kun Jia ${ }^{1}$, Tripta
} Rughwani $^{1}$, Qi Wang ${ }^{1}$, Sven Kroener ${ }^{2}$, Lan Guo ${ }^{1 *}$, Heng Du ${ }^{1^{*}}$

1) Department of Biological Sciences, University of Texas at Dallas. Richardson, TX, 75080

2) School of Behavioral and Brain Sciences, University of Texas at Dallas. Richardson, TX, 75080

\# Contribute equally

Esha Gauba: egauba@stanford.edu

Shaomei Sui: suizia5@163.com

Jing Tian: jxt131830@utdallas.edu

Christopher Driskill: cmd120130@utdallas.edu

Chunxiao Yu: Chunxiao.Yu@utdallas.edu

Kun Jia: Kun.Jia@utdallas.edu

Tripta Rughwani: Tripta.Rughwani@utdallas.edu

Qi Wang: wangqi29@126.com

Sven Kroener: kroener@utdallas.edu

*Corresponding authors:

Lan Guo MD PhD

Assistant Research Professor

Department of Biological Sciences

The University of Texas at Dallas

Richardson, TX, 75080

lan.guo@utdallas.edu

9728833531

Heng Du MD PhD

Associate Professor

Department of Biological Sciences

The University of Texas at Dallas

Richardson, TX, 75080 
Heng.du@utdallas.edu

9728833532

\section{ABSTRACT}

Background Oligomycin-sensitivity conferring protein (OSCP) is a critical subunit of mitochondrial F1Fo ATP synthase. OSCP dysfunction has been observed in Alzheimer's disease (AD) brains and a mouse model with AD-like brain amyloidosis (5xFAD mice). However, whether OSCP dysfunction constitutes a key mitochondrial defect contributing to synaptic injury in AD-related conditions has not been comprehensively investigated.

Methods Here, we used a 5xFAD mouse model with OSCP overexpression in neurons (Thy-1 OSCP/5xFAD mice) and cultured neurons from OSCP overexpressing pups in the study. We performed biochemical, immunohistochemical, live cell imaging and electrophysiological as well as behavioral analyses.

Results We found that preserved OSCP expression with reduced interaction of amyloid beta (A $\beta$ ) with membrane-bound OSCP in Thy- 1 OSCP/5xFAD mice. OSCP overexpression also alleviated F1Fo ATP synthase deregulation and preserved mitochondrial function. Moreover, OSCP modulation conferred resistance to $\mathrm{A} \beta$-mediated defects in axonal mitochondrial dynamics \& motility. Consistent with protected neuronal mitochondrial function, OSCP overexpression ameliorated hippocampal synaptic injury in $5 \mathrm{xFAD}$ mice as demonstrated by preserved synaptic density and synaptic transmission, reduced complement-dependent synapse elimination, leading to improved spatial learning and memory. 
Conclusion Our findings show the consequences of OSCP dysfunction in the development of synaptic stress in AD-related conditions and they implicate OSCP modulation as a potential therapeutic strategy.

\section{KEYWORDS}

Oligomycin sensitivity-conferring protein, mitochondrial F1Fo ATP synthase, synaptic injury, amyloid beta, Alzheimer's disease

\section{BACKGROUND}

Alzheimer's disease (AD) is a chronic neurodegenerative disorder characterized by insidious onset and progressive cognitive decline (1). Effective treatments that benefit patients with $\mathrm{AD}$ are still lacking. Although amyloid beta $(\mathrm{A} \beta)$ deposition is a defining pathology of $\mathrm{AD}$ (2), recent clinical failures challenged the effectiveness of interventions against $\mathrm{A} \beta$ per se for the treatment of this neurological disorder $(3,4)$, prompting a reappraisal of alternative therapeutic pathways. Previous observations of brain hypometabolism, oxidative damages, and energy deficiency support the mitochondrial cascade hypothesis of AD (5-7). Although it remains unclear whether mitochondrial dysfunction is a primary cause of $\mathrm{AD}$, cumulative evidence supports a model in which mitochondrial defects confer susceptibility to A $\beta$-induced synaptotoxicity and neuronal stress, leading to irreversible damages and cognitive impairment (8). For this reason, mitochondrial dysfunctions that contribute to AD-related neuronal perturbations might provide novel therapeutic targets to prevent neurodegenerative processes that stem from $A \beta$ toxicity.

Reduced energy production due to compromised oxidative phosphorylation (OXPHOS) is a prominent feature of mitochondrial pathology in $\mathrm{AD}$ neurons. In addition to defects in the 
mitochondrial electron transport chain (ETC), deregulation of F1Fo ATP synthase, the key enzyme for mitochondrial OXPHOS $(9,10)$, has been linked to lowered OXPHOS efficacy in cognitive aging (11) and $\mathrm{AD}(12,13)$. Oligomycin sensitivity-conferring protein (OSCP), a component of the F1Fo ATP synthase peripheral stalk, is a critical subunit for the structural stability and function of this enzyme $(14,15)$. Patients with AD show loss of OSCP and OSCP/A $\beta$ complexation in their brains (12). These OSCP abnormalities are believed to underlie F1Fo ATP synthase deregulation and they contribute to neuronal mitochondrial dysfunction in AD-relevant settings (11-13). Given the pivotal role of mitochondrial fitness in synaptic physiology (16-18), the deleterious impact of OSCP changes on mitochondrial function implicates an OSCP-related mechanism of synaptic failure in AD. We therefore hypothesized that OSCP perturbations could be targeted to mitigate $A \beta$-induced synaptic stress.

To experimentally address this idea, we overexpressed OSCP in neurons under the control of the Thy-1 promoter in 5xFAD mice (Thy-1 OSCP/5xFAD mice). This modulation restored OSCP expression without affecting other major F1Fo ATP synthase subunits in 5xFAD mice. Moreover, the complexation of $\mathrm{A} \beta$ with inner mitochondrial membrane (IMM)-bound OSCP was attenuated by OSCP overexpression. In addition to its beneficial effect on F1Fo ATP synthase activity and mitochondrial bioenergetics in 5xFAD mice, OSCP overexpression prevented axonal mitochondrial dynamics and motility from $A \beta$ toxicity. Importantly, Thy-1 $\mathrm{OSCP} / 5 \mathrm{xFAD}$ mice exhibited normal synaptic density and synaptic transmission, as well as reduced synapse pruning, and preserved cognitive performances. Our findings suggest a role of neuronal OSCP dysfunction in the pathogenesis of synaptic degeneration in AD-related pathological settings and suggest novel pathways in the development of treatments for AD.

\section{METHODS AND MATERIALS}


Mice. Animal studies were approved and performed under the guidelines of the University of Texas at Dallas (UTD) Institutional Animal Care and Use Committee (IACUC) and National Institutes of Health (NIH). In order to generate Thy-1 OSCP transgenic mice, a recombinant vector was designed to place human OSCP cDNA (Gene Name: ATP5O. NCBI Gene ID: 539) under control of mouse Thyl gene promoter [gift from Joshua R. Sanes (54): Addgene plasmid 20736]. This construct was microinjected into pronuclei of single-cell B6SJLF1/J hybrid embryos by the staff at the transgenic core at UT Southwestern Medical Center. Founder transgenic mice were identified by PCR and confirmed by Southern blot analysis. Transgenic founders were backcrossed to B6SJLF1/J background.

Thy-1 OSCP mice were crossed with 5xFAD mice (B6SJL-Tg(APP-SwFlLon, PSEN1*M146L*L286V)6799Vas/Mmjax) which were originally obtained from Jackson Laboratory to generate litters including non-transgenic (nonTg), 5xFAD, Thy-1 OSCP, and Thy1 OSCP/5xFAD mouse. Genotypes of animals were confirmed using PCR and/or amyloid plaques staining. The minimum number of mice needed was determined by power calculation based on our previous results. To mimic mild cognitive impairment (MCI) and later stage AD, respectively, the studies were performed using 4-5 and 9-10-month-old mice. Both male and female mice were used.

Immunoblotting. Samples were prepared as previously described (12). Mitochondria samples were prepared and lysed in RIPA lysis buffer (50 mM Tris- $\mathrm{HCl}$, $\mathrm{pH} 8.0$, with $150 \mathrm{mM}$ sodium chloride, $1.0 \%$ NP-40, $0.5 \%$ sodium deoxycholate, and $0.1 \%$ sodium dodecyl sulfate). Neurons were collected in sample loading buffer $(50 \mathrm{mM}$ Tris- $\mathrm{HCl} \mathrm{pH}$ 6.8, 2\% SDS, $10 \%$ glycerol, $1 \% \beta-$ mercaptoethanol, $12.5 \mathrm{mM}$ EDTA and $0.02 \%$ bromophenol blue) with Protease Inhibitor Cocktail Set V (Millipore) and phosphatase inhibitors including Sodium Fluoride (Sigma-Aldrich) and 
Sodium Orthovanadate (Sigma-Aldrich). Proteins were separated on a 12\% Bis-tris gel (NuPage, Life Technologies) and transferred to PVDF membrane (ImmunBlot membrane, BioRad) at $120 \mathrm{~V}$ for 90 minutes. Membranes were blocked with 5\% milk (Labscientific Inc) for 1 hour at room temperature followed by primary antibody incubation for overnight at $4^{\circ} \mathrm{C}$. The following day, membranes were washed with $1 \times$ TBS and incubated with secondary antibody at the appropriate concentration. The following antibodies were used in this study: anti-A $\beta$ (CST, 1:1000), anti-Tom40 (Santa Cruz, 1:500), anti-a subunit (Proteintech, 1:5000), anti-b subunit (Santa Cruz, 1:500), anti-c subunit (Abcam, 1:3000), anti-OSCP (Santa Cruz, 1:5000), anti- $\alpha$ subunit (Santa Cruz, 1:5000), anti- $\beta$ subunit (Santa Cruz, 1:5000), anti- $\gamma$ subunit (Santa Cruz, 1:5000), anti-Opa1 (BD Biosciences, 612606, 1:1000), anti-Mitofusin-2 (Mfn2) (Cell Signaling Technology, 9482S, 1:3000), anti-Dynamin Like Protein-1 (Dlp1) (BD Biosciences, 611112, 1:2000), anti-phospho-Dlp1 (Ser616) (Cell Signaling Technology, 3455), anti-C1q (Dako, A0136, 1:1000), HRP conjugated goat anti-mouse IgG (H+L) (Thermo Fisher Scientific, 626520, 1:2000), and HRP conjugated goat anti-rabbit $\operatorname{IgG}(\mathrm{H}+\mathrm{L})$ (Thermo Fisher Scientific, 656120, 1:2000). Images were collected on a Bio-Rad Chemidoc Imaging System. Image J (National Institutes of Health) was used to analyze the blots and to quantify protein signal intensity.

Synaptosome isolation. Synaptosomes were isolated as previously described $(12,13)$. In brief, brain tissues were homogenized in ice cold buffer [225 mM mannitol, $75 \mathrm{mM}$ sucrose, $2 \mathrm{mM}$ $\mathrm{K}_{2} \mathrm{PO}_{4}, 0.1 \%$ BSA, $5 \mathrm{mM}$ Hepes, and $1 \mathrm{mM}$ EGTA ( $\mathrm{pH}$ 7.2)] using a Wheaton Dounce homogenizer. The resultant homogenate was centrifuged at $1,300 \times \mathrm{g}$ for 3 minutes to remove blood and cell debris in the pellet, and the supernatant was layered on a discontinuous Percoll density gradient consisting of $15 \%, 23 \%$, and $40 \%$ (vol/ vol) Percoll. After centrifugation at 
$34,000 \times \mathrm{g}$ for 5 minutes, synaptosomes were collected from the interface between $15 \%$ and $23 \%$ Percoll.

Brain mitochondria isolation. Brain mitochondria were prepared as previously described (13). Cortices were dissected from mouse brain and homogenized in ice cold isolation buffer [ $225 \mathrm{mM}$ mannitol, $75 \mathrm{mM}$ sucrose, $2 \mathrm{mM}$ K2PO4, 0.1\% BSA, $5 \mathrm{mM}$ Hepes, and $1 \mathrm{mM}$ EGTA (pH 7.2)] with a Wheaton Dounce homogenizer. After a centrifugation at $1,300 \times \mathrm{g}$ for 5 minutes to remove blood and cell debris, the supernatant was layered on 15\% Percoll (GE) and centrifuged at $125,00 \times \mathrm{g}$ for 10 minutes. The pellets were collected and resuspended in isolation buffer with $0.02 \%$ Digitonin (Sigma-Aldrich), followed by centrifugation at $8,000 \times \mathrm{g}$ for another 10 minutes. The pellets were then washed with an additional centrifugation step in ice-cold isolation buffer without EGTA for experiments. Protein concentrations were measured using Bradford assay for protein detection (BioRad).

Mitochondrial membrane isolation. The isolated mitochondria were broken in isolation buffer containing 5\% Digitonin on ice for 15 minutes followed by sonication on ice for 20 minutes. The mitochondrial membranes were pelleted by centrifugation at $130,000 \times \mathrm{g}$ for 1 hour at $4^{\circ} \mathrm{C}$ using a Beckman Coulter ultracentrifuge (Optima XPN-90).

Co-immunoprecipitation (Co-IP) of OSCP and Aß. Co-immunoprecipitation was performed using $0.5 \mathrm{mg}$ of mitochondria or mitochondrial membrane-rich fractions. The indicated fractions were lysed using Lysis buffer (50mM Tris-HCl, $150 \mathrm{mM} \mathrm{NaCl}$, 1mM EDTA, 0.5\% NP-40, 5\% glycerol and $1 \mathrm{X}$ protease inhibitor) by incubation on ice for 30 minutes, then subjected to five freeze-thaw cycles, followed by centrifugation at $12,500 \times \mathrm{g}$ for 10 minutes at $4{ }^{\circ} \mathrm{C}$. The Supernatants were incubated with anti-OSCP (Santa Cruz, $0.5 \mu \mathrm{g} \mathrm{IgG/100 \mu g}$ protein) to form 
immune-complexes at $4^{\circ} \mathrm{C}$ overnight. Pre-immune IgG was used at the same concentration to determine protein-protein interaction specificity. The immune-complexes were precipitated by using pre-cleaned $\mathrm{A} / \mathrm{G}$ beads (Pierce), followed by immunoblotting with antibody to $\mathrm{A} \beta$ (CST, 1:1000) to identify OSCP-A $\beta$ interaction.

Duolink proximity ligation assay (PLA). The interaction between OSCP and A $\beta$ in mouse brain slices was detected using Duolink In Situ PLA detection kits (Sigma-Aldrich) following the manufacturer's instruction. Mouse anti-OSCP (Santa Cruz Biotechnologies, 1: 200) and rabbit anti-A $\beta$ (CST, 1:1000) were use as primary antibodies. Anti-Rabbit PLUS (SigmaAldrich, \#DUO92002) and anti-Mouse MINUS (Sigma-Aldrich, \#DUO92004) were applied as Duolink in Situ PLA Probes. Images were collected on a Nikon confocal microscope. The images were converted to three dimensional images by using the Nikon NIS Elements Advanced Research Software "3D reconstruction" module for analysis. The number of PLA-positive dots was counted, and divided by total volume of the image stack.

F1FO ATP synthase enzymatic activity assay. F1FO ATP synthase enzyme activity was measured spectrophotometrically using NADH-linked ATP regenerating system. Mitochondria were resuspended in activity assay buffer $[100 \mathrm{mM}$ Tris $\mathrm{HCl}(\mathrm{pH} 7.4), 2 \mathrm{mM} \mathrm{MgCl} 2,50 \mathrm{mM}$ $\mathrm{KCl}, 0.2 \mathrm{mM}$ EDTA, $0.23 \mathrm{mM} \mathrm{NADH}$, and $1 \mathrm{mM}$ phosphoenolpyruvate). The reaction was triggered by adding $0.4 \mathrm{M} \mathrm{Mg-ATP}$ and recorded on a spectrophotometer at OD $340 \mathrm{~nm}$ for a total of 10 minutes.

F1FO ATP synthase coupling assay. Enzyme coupling assay was performed by incubating the mitochondria with Oligomycin A at the mentioned concentration for 15 minutes at room temperature before measuring the enzymatic activity of F1FO ATP synthase (12). 
Oxygen Consumption Assay. Oxygen consumption from mitochondria was measured polarographically using a temperature-regulated Clark-type oxygen electrode (Oxytherm, Hansatech). Freshly isolated brain mitochondria were added to the magnetically stirred chamber and energized with $5 \mathrm{mM}$ Glutamate/Malate (Sigma). To measure the respiration control ratio (RCR), $300 \mu \mathrm{M}$ ADP was added and state III was measured. Once exogenous ADP is used up mitochondria enter state IV respiration. RCR was measured as a ratio of state III/state IV. The ADP:O ratio was calculated as the ratio of ADP conversion rate at state III respiration to two fold of oxygen consumption rate at state III respiration (55).

ATP measurement. ATP synthesis from freshly isolated mitochondria or cultured neurons was measured using a Luminescence ATP detection Assay Kit (Abcam) according to manufacturer's instructions. Luminescence was detected using a microplate reader (synergy Mx., Biotek) controlled by Gen5 software. Standard curve was prepared using ATP as substrate and luminescence readings were expressed in fold change.

Mitochondrial swelling assay. Mitochondria swelling was performed on freshly isolated mitochondria. Neuronal mitochondria were suspended in $0.5 \mathrm{~mL}$ of swelling assay buffer [50 $\mu \mathrm{g}$ of mitochondrial protein, $150 \mathrm{mM} \mathrm{KCl}, 5 \mathrm{mM}$ HEPES, $2 \mathrm{mM} \mathrm{K}_{2} \mathrm{HPO}_{4}, 5 \mathrm{mM}$ glutamate $(\mathrm{pH}$ 7.2)]. Mitochondrial swelling was triggered by the addition of calcium ( $500 \mathrm{nmol} / \mathrm{mg}$ of protein). Swelling was observed by immediately and continuously recording changes in OD values at 540 nm by using a spectrophotometer (Ultrospect 2100, Amersham Biosciences).

Immunocytochemistry. Mouse brains were dissected and immediately fixed in 4\% paraformaldehyde (PFA) (Sigma-Aldrich) for $24-26$ hours at $4{ }^{\circ} \mathrm{C}$. The frozen tissue sections were prepared as previously described (56). Primary cultured neurons at DIV14 cultured on Lab- 
Tek chamber slides were fixed in $4 \%$ PFA for 30 minutes at $37^{\circ} \mathrm{C}$. The slices or neurons were blocked with blocking buffer (5\% goat or donkey serum, $0.3 \%$ Triton X-100 in PBS, $\mathrm{pH} 7.4$ ) for 1 hour, then incubated with primary antibodies at room temperature overnight. The following primary antibodies were used: mouse anti-PSD95 antibody (Cell Signaling Technology, 36233, 1:400), guinea pig anti-vesicular glutamate transporter 1 (vGlut1, Synaptic Systems, 135304, 1:400), anti-Ibal (Abcam, ab5076, 1:600), rabbit anti-synaptophysin (Cell Signaling, Technology, 5461, 1:500), and mouse anti-C1q (Abcam, ab71940, 1:300). After washing with PBS, the slices or neurons were then probed with appropriate cross-adsorbed secondary antibodies conjugated to Alexa Fluor 488, Alexa Fluor 594, or Alexa Fluor 647 (Thermo Fisher Scientific, 1:500). Images were collected under a Nikon confocal microscope followed by threedimensional reconstruction and analysis using Nikon NIS Elements Advanced Research Software. The synapses were defined by colocalization of vGlut1 and PSD95 which was analyzed by using the "AND" operation in the "binary operation" dialog of NIS element software to overlap two binary layers. The number of synapses was counted, and divided by total volume of the image stack. C1q-tagged synapses were defined by colocalization of C1q and synaptophysin and calculated as the ratio of C1q-colocalized synaptophysin to total synaptophysin (volume/volume). Synaptic pruning was examined through co-staining of Iba1 and synaptophysin and calculated as the ratio of engulfed synaptophysin by microglia to total synaptophysin (volume/volume).

A $\beta$ immunostaining. PFA-fixed frozen brains were sectioned and used for $A \beta$ staining to determine the levels of plaque deposition. After blocking in PBS containing 5\% goat serum and $0.3 \%$ Triton $\mathrm{X}-100$, the free-floating brain sections were incubated overnight at room temperature with anti-A $\beta$ (CST, 1:1000). After washing with PBS, the slices were probed with 
cross-adsorbed secondary antibodies conjugated to Alexa Fluor 488 (Thermo Fisher Scientific, 1:500). Neurons were identified by the staining of NISSL blue (Sigma). Images were collected on a Nikon inverted fluorescence microscope. Nikon NIS Elements Advanced Research Software was used for image analysis. Plaque-occupied area was calculated as a percentage ratio of the area of the plaque to that of the cortex.

Electrophysiological measurement. For local field potential experiments (LFPs), mice were anesthetized with isoflurane and decapitated. Brains were rapidly extracted and transverse sections $(350 \mu \mathrm{m})$ of the hippocampus were cut on a vibratome (VT1200S, Leica) in ice-cold oxygenated $\left(95 \% \mathrm{O}_{2}, 5 \% \mathrm{CO}_{2}\right)$ low sodium ACSF containing the following: $110 \mathrm{mM}$ choline (Sigma-Aldrich), $25 \mathrm{mM} \mathrm{NaHCO}_{3}$ (Fisher Scientific), $1.25 \mathrm{mM} \mathrm{NaH}_{2} \mathrm{PO}_{4}$ (Fisher Scientific), 2.5 $\mathrm{mM} \mathrm{KCl}$ (Sigma-Aldrich), $7 \mathrm{mM} \mathrm{MgCl} 2$ (Sigma-Aldrich), $0.5 \mathrm{mM} \mathrm{CaCl}$ (Sigma-Aldrich), 10 $\mathrm{mM}$ dextrose (Fisher Scientific), 1.3 mM L-ascorbic acid (Fisher Scientific), and $2.4 \mathrm{mM}$ sodium pyruvate (Sigma-Aldrich). Slices were incubated for at least 1 hour in normal recording ACSF consisting of: $126 \mathrm{mM} \mathrm{NaCl}$ (Fisher Scientific), $25 \mathrm{mM} \mathrm{NaHCO}_{3}, 1.25 \mathrm{mM} \mathrm{NaH}_{2} \mathrm{PO}_{4}, 2.5 \mathrm{mM}$ $\mathrm{KCl}, 1.3 \mathrm{mM} \mathrm{MgCl}_{2}, 2 \mathrm{mM} \mathrm{CaCl} 2,10 \mathrm{mM}$ dextrose, $2.4 \mathrm{mM}$ sodium pyruvate, and $1.3 \mathrm{mM} \mathrm{L}-$ ascorbic acid, bubbled with $95 \% \mathrm{O}_{2} / 5 \% \mathrm{CO}_{2}$. Slices were allowed to rest for 30 minutes after being transferred to the recording chamber. Recordings of LFPs were performed on an Axon Multiclamp 700B amplifier (Molecular Devices), and data were acquired and analyzed using AxoGraph X (AxoGraph Scientific). A tungsten concentric bipolar microelectrode (World Precision Instruments), and a recording glass electrode (1.5 M 2$)$ filled with recording ACSF were placed approximately $200 \mu \mathrm{m}$ apart in the Schaffer collateral-commissural pathway in the CA1 region of the hippocampus. Pulses were delivered in 30 second intervals. Before baseline recordings commenced, input-output curves were taken, using 25-150 $\mu \mathrm{A}$ stimulation currents in 
$25 \mu \mathrm{A}$ steps. Three sweeps were sampled at each stimulation intensity and averaged to represent the voltage response at each step. Recordings of baseline responses lasted for at least 40 minutes. Stimulation intensity was set to approximately $40 \%$ of the minimum intensity required to evoke the maximum response (based on the input-output curve). The stimulation intensity was kept consistent throughout the duration of the experiment. LTP was induced using a theta burst stimulation (TBS) protocol consisting of 10 bursts (5 pulses at $100 \mathrm{~Hz}$ ) repeated at $5 \mathrm{~Hz}$, delivered two times 30 seconds apart. After LTP induction, LFPs were recorded for an additional 60 minutes. The slope of the EPSP was measured in Axograph and sweeps were averaged in bins of 5 for both the baseline and post LTP induction periods. Changes in LFPs are expressed as percentage change from the averaged baseline values.

ELISA assay for $A \boldsymbol{\beta}$ measurement. $A \beta$ amounts in mouse cortical or mitochondria samples were measured by using human $A \beta 40$ and $A \beta 42$ ELISA kits (Thermo Fisher Scientific, KHB3481 for A 340 and KHB3441 for A 342 ) following the manufacturer's instructions. Tissues were homogenized thoroughly with cold $5 \mathrm{M}$ guanidine $\mathrm{HCl} / 50 \mathrm{mM}$ Tris $\mathrm{HCl}$. The homogenates were incubated at room temperature for 4 hours. The samples were diluted with cold reaction buffer (Dulbecco's phosphate buffered saline with 5\% BSA and 1X protease inhibitor cocktail) and centrifuged at $16,000 \times \mathrm{g}$ for 20 minutes at $4^{\circ} \mathrm{C}$. The supernatants were diluted with standard diluent buffer provided in the kit and quantified by ELISA kits. A $\beta$ amounts were normalized to total protein content in the samples.

Primary neuron culture. Primary neurons were cultured from nonTg and OSCP OE pups as previously described (12). In brief, brain tissues were dissected from day 0 pups and immediately put in cold Hank's balanced salt solution (HBSS, Sigma-Aldrich), dissociated with $0.05 \%$ trypsin (Sigma-Aldrich) at $37^{\circ} \mathrm{C}$ for 15 minutes followed by $10-15$ times trituration. Cells were then 
passed through a $40 \mu \mathrm{m}$ mesh cell strainer (Fisher brand) and centrifuged for 5 minutes at $200 \times$ g. The pellet was gently resuspended in neuron culture medium (Neurobasal A with 2\% B27 supplement and $0.5 \mathrm{mM} \mathrm{L-glutamine,} \mathrm{Gibco)} \mathrm{and} \mathrm{plated} \mathrm{on} \mathrm{poly-D-lysine} \mathrm{(Sigma-Aldrich)}$ coated culture plates (Corning) or chamber slides (Nunc) with an appropriate density (12). 10 $\mu \mathrm{M}$ 5-fluoro-2' -deoxyuridine (Sigma-Aldrich) and Uridine (Sigma-Aldrich) was added to the neuron cultures to inhibit non-neuronal cell proliferation. Neurons were cultured to 14 days in vitro (DIV) for experiment.

A $\beta 42$ and scrambled Aß42 preparation. The $\mathrm{A} \beta 42$ oligomer (GenicBio) was prepared as we previously described (16). A $\beta 421-1$ peptide (Anaspec) was prepared following the manufacturer's instructions.

Axonal mitochondrial length and volume measurement. Neurons were infected by lentivirusexpressing mitochondrial targeted DsRed (mitoDsRed) at DIV7. At DIV13, the neurons were treated with vehicle, $1 \mu \mathrm{M} \mathrm{A} \beta 42$ oligomer or $1 \mu \mathrm{M}$ scrambled $\mathrm{A} \beta$ for 24 hours, respectively and imaged. A process two to three times longer than other processes stemming from the soma was considered to be an axon as previously described (16). The images were collected on a Nikon inverted fluorescent microscope with on-stage incubator $\left(37^{\circ} \mathrm{C}, 5 \% \mathrm{CO}_{2}\right)$, and a fluorescent particle with a clear border was identified as a mitochondrion. The axonal mitochondrial length was analyzed by using Nikon NIS Elements Advanced Research software. For axonal mitochondrial volume measurement, the neurons were fixed with $4 \%$ paraformaldehyde (SigmaAldrich) for 30min. After blocking in 5\% BSA and 0.2\% Triton X-100 (Sigma-Aldrich) for 1 hour at room temperature, the neurons were stained with rabbit anti-Microtubule associated protein 2 (MAP2) (Cell Signaling Technology, 8707) to determine dendrites and mouse antiTau-1 (Cell Signaling Technology, 4019) to determine axons followed by Alexa Fluor 488 
conjugated goat anti-rabbit $\operatorname{IgG}(\mathrm{H}+\mathrm{L})$ secondary antibody (Thermo Fisher Scientific, R37116) and Alexa Fluor 647 conjugated goat anti-mouse $\operatorname{IgG}(\mathrm{H}+\mathrm{L})$ highly cross-adsorbed secondary antibody (Thermo Fisher Scientific, A32728), respectively. Images were collected under a Nikon confocal microscope followed by three-dimensional reconstruction and analysis was done using Nikon NIS Elements Advanced Research Software.

Axonal mitochondria movement recording. The neurons were cultured on glass bottom microplates (Corning, 4580) and treated using the same method as axonal mitochondrial length and volume measurement. Time-lapse images were captured every 5 seconds for a period of 2 minutes using a Nikon inverted fluorescent microscope with CFI plan fluor $40 \times$ oil immersion objective lens and on-stage incubator $\left(37^{\circ} \mathrm{C}, 5 \% \mathrm{CO} 2\right)$. A mitochondrion was considered to be non-mobile if it remained stationary for the entire recording period (57). A movement was counted only if there was a displacement more than the length of the mitochondrion. The direction of movement was determined as stationary, anterograde, or retrograde by comparing the displacement between the initial and final positions relative to the cell body. Mitochondrial movement toward the distal end of an axon was considered to be anterograde, whereas movement toward the proximal end was considered to be retrograde. Kymographs were generated using maximum intensity projections to demonstrate the overall movement traces of mitochondria during the recording period (57).

Mitochondrial membrane potential assay. Neurons were incubated with $200 \mathrm{nM}$ TMRM (Sigma-Aldrich) for 30 minutes in an incubator $\left(37^{\circ} \mathrm{C}, 5 \% \mathrm{CO}_{2}\right)$. Thereafter, the neurons were washed with pre-warmed neuron culture medium followed by another 15-minute incubation. TMRM staining was imaged on a Nikon inverted fluorescent microscope with on-stage incubator 
$\left(37^{\circ} \mathrm{C}, 5 \% \mathrm{CO}_{2}\right)$. TMRM intensity was analyzed using Nikon NIS Elements Advanced Research software (12).

Mitochondrial superoxide assay for cultured neurons. Cultured neurons were exposed to 2 $\mu \mathrm{M}$ MitoSox Red (Thermo Fisher Scientific, M36008) and $200 \mathrm{nM}$ MitoTracker green (Thermo Fisher Scientific, M7514) for 45 minutes in an incubator $\left(37^{\circ} \mathrm{C}, 5 \% \mathrm{CO}_{2}\right)$ followed by washing using pre-warmed neuron culture medium. The live neuron nucleus was identified of Hoechst 33342 (Fisher Scientific) staining at a concentration of $1 \mu \mathrm{g} / \mathrm{ml}$. The images were collected on a Nikon inverted fluorescent microscope with on-stage incubator $\left(37^{\circ} \mathrm{C}, 5 \% \mathrm{CO}_{2}\right)$ and analyzed using Nikon NIS Elements Advanced Research software.

4-hydroxynonenal (4-HNE) staining. Cultured neurons with the expression of mitoDsRed were fixed in $4 \%$ paraformaldehyde for 30 minutes in an incubator $\left(37^{\circ} \mathrm{C}, 5 \% \mathrm{CO}_{2}\right)$. After incubation in blocking buffer ( $5 \%$ goat serum, $0.3 \%$ Triton, PBS), the neurons were incubated with rabbit anti-4-HNE (Abcam, ab46545) at $4^{\circ} \mathrm{C}$ overnight, followed by incubation with Alexa Fluor 488 conjugated goat anti-rabbit $\operatorname{IgG}(\mathrm{H}+\mathrm{L})$ (Thermo Fisher Scientific, R37116) for 1 hour at room temperature. The images were collected on a Nikon confocal microscope and analyzed using Nikon NIS Elements Advanced Research Software.

Behavioral Studies. To study the spatial learning and reference memory in non-AD and AD mice, the Morris water maze task was performed as previously described. Mice were trained to swim and locate a hidden platform in a water-filled tank for 11 days. After 4 sets of training per day, each set lasting for 30 seconds, mice were subjected to a probe test in which the platform was removed. The goal is to identify the quadrant of the platform and measure the passes at the 
specified location of the platform. HVS Image 2015 software (HVS Image) was used to track and monitor the behavioral testing of the animals.

Statistical analysis. Statistical comparisons were performed using GraphPad Prism 7 software. One-way or two-way ANOVA followed by Bonferroni post hoc analysis, or unpaired two-way Student's $t$ test were applied in data analysis. Pearson's correlation coefficient was used for correlation testing. Numbers of replicates and $P$ values are stated in each figure legend. All data were expressed as the mean \pm s.e.m. except for the box plots which were shown as maximum, median and minimum. Significance was concluded when the $P$ value was less than 0.05 . Significance was indicated by symbols including * $(P<0.05),{ }^{* *}(P<0.010),{ }^{* * *}(P<0.001)$, NS (not significant) denotes $P>0.05$.

\section{RESULTS}

\section{OSCP dysfunction is reduced in Thy-1 OSCP/5xFAD mice.}

To restore neuronal OSCP with minimal side effects due to excess protein expression, we selected a line of Thy-1 OSCP mice with moderate ( 1.4-1.5 fold) OSCP upregulation in neurons (Fig. 1A\&B) and generated Thy-1 OSCP/5xFAD mice by crossbreeding. To examine the consequence of OSCP overexpression, we measured OSCP expression in cortical mitochondrial fractions via immunoblotting. Brain mitochondria were purified from the neocortex of gender- and age-matched non-transgenic (nonTg), 5xFAD, Thy-1 OSCP, and Thy-1 OSCP/5xFAD mice at 4-5 and 9-10 months old. These age ranges were selected based on our previous observations of OSCP changes, mitochondrial dysfunction, synaptic injury, and cognitive impairment in 5xFAD mice $(12,13,19)$. Densitometric analysis of the immunoreactive bands showed decreased OSCP expression in 5xFAD mice at both ages, which was reversed by 
OSCP overexpression (Fig. 1A\&B). To determine whether OSCP overexpression affects the expression of other major subunits of F1Fo ATP synthase, we further examined the expression of $\alpha, \beta, \gamma, \mathrm{a}, \mathrm{b}$, and $\mathrm{c}$ subunits. Immunoblotting data showed no change in the content of these subunits across all four groups of mice at the tested ages (Fig. 1A\&B). The results confirm selective OSCP vulnerability in AD-relevant pathological settings $(12,13)$ and the restoration of OSCP expression in Thy-1 OSCP/5xFAD mice.

OSCP interacts with mitochondrial $\mathrm{A} \beta(12,13)$. OSCP upregulation may therefore promote the formation of $\mathrm{OSCP} / \mathrm{A} \beta$ complexes. As expected, further assays for in situ $\mathrm{OSCP} / \mathrm{A} \beta$ interaction, and by proxy, the positive dots of proximity ligation assays (PLA), showed agedependent increases in OSCP/A $\beta$ complexation in neocortical neurons in Thy- 1 OSCP/5xFAD mice when compared with cells from non-OSCP-modulated 5xFAD littermates (Fig. 1C1\&2). Similar results were seen with neuronal mitochondrial fractions from 9-10 months old 5xFAD and Thy-1 OSCP/5xFAD mice when using co-immunoprecipitation (co-IP) (Fig. 1D1\&2). Further co-IP for OSCP and $\mathrm{A} \beta$ complexation using mitochondrial membrane-rich fractions also showed decreased mitochondrial membrane-bound OSCP/A $\beta$ complexes (Fig. 1D1\&2). Of note, OSCP overexpression did not affect A $\beta$ plaque deposition (Supplementary Fig. 1A\&B) or concentrations of soluble A $\beta 1-40$ or A $\beta 1-42$ in either cortex homogenates (Supplementary Fig. 2A\&B) or mitochondrial fractions (Supplementary Fig. 2C\&D) from 5xFAD mice. Because F1Fo ATP synthase is located on the inner mitochondrial membrane, these results seem to suggest decreased $\mathrm{A} \beta$ interaction with OSCP that is integrated in F1Fo ATP synthase. Such an effect is, at least in part, due to A $\beta$ neutralization by increased "free" OSCP in the mitochondrial matrix. If so, we would expect to see preserved mitochondrial F1Fo ATP synthase activity and improved mitochondrial function in Thy-1 OSCP/5xFAD mice. 


\section{Mitochondrial function is preserved in Thy-1 OSCP/5xFAD mice.}

To determine the protective effect of OSCP overexpression on mitochondrial function, we prepared brain mitochondria from gender- and age-matched nonTg, Thy-1 OSCP, 5xFAD, and Thy-1 OSCP/5xFAD mice at 4-5 and 9-10 months old and subjected them to assays for F1Fo ATP synthase function. Consistent with our previous observation $(12,13)$, mitochondria from 5xFAD mice demonstrated decreased F1Fo ATP synthase activity in an age-dependent manner, which was ameliorated in OSCP-overexpressing 5xFAD mice (Fig. 2A1\&2). Next, we examined F1Fo ATP synthase oligomycin sensitivity to reflect F1Fo complex proton-flow coupling (12, 20). F1Fo ATP synthase oligomycin sensitivity was protected in 5xFAD mice through OSCP overexpression (Fig. 2B1, 2), suggesting that OSCP overexpression prevents F1Fo complex function and integrity in A $\beta$-enriched environments. Notably, Thy-1 OSCP mice did not demonstrate changes in F1Fo ATP synthase when compared with their nonTg littermates (Fig.

2A\&B). Because OSCP overexpression had no effect on the content of other major F1Fo ATP synthase subunits, this suggests that OSCP upregulation has minimal genotypic effects on baseline levels of F1Fo ATP synthase function.

To determine whether preserving F1Fo ATP synthase function via OSCP restoration benefits mitochondria in 5xFAD mice, we examined mitochondrial function in four groups of mice at 4-5 and 9-10 months old. When compared with their age- and gender-matched nonTg counterparts, 5xFAD mice exhibited an age-dependent decrease in mitochondrial respiration control ratio (RCR) (Fig. 2C1\&2) along with lessened ATP production (Fig. 2D1\&2) and a decreased ADP:O ratio (Fig. 2E1\&2). In contrast, these 5xFAD genotypic changes were ameliorated in Thy-1 OSCP/5xFAD mice (Fig. 2C-E), indicating a strong correlation of OSCP aberrations with A $\beta$-perturbed mitochondrial bioenergetics. Because F1Fo ATP synthase has 
been linked to mitochondrial $\mathrm{Ca}^{2+}$ regulation $(12,13,21-23)$, we next performed mitochondrial swelling assays, and found that OSCP-overexpressing 5xFAD mice showed less mitochondrial swelling in response to $\mathrm{Ca}^{2+}$ stimulation (Fig. 2F1\&2). These results indicate that OSCP overexpression protects against the $\mathrm{A} \beta$-induced decrease in mitochondrial $\mathrm{Ca}^{2+}$ handling capacity. These findings, along with preserved F1Fo ATP synthase function, are in line with the detrimental role of F1Fo ATP synthase dysfunction in mitochondrial permeability $(12,13,21$ 23). Thy-1 OSCP mice did not demonstrate the same changes as their nonTg littermates at either of the ages tested (Fig. 2A-F), indicating little genotypic effect of OSCP overexpression. Taken together, these results underline the contribution of OSCP dysfunction to mitochondrial deficits in AD-related pathological settings and they demonstrate that OSCP restoration protects mitochondrial function against $A \beta$ toxicity.

\section{OSCP modulation protects axonal mitochondrial dynamics and motility against A $\beta$ toxicity.}

Neuronal mitochondria are dynamic organelles $(24,25)$. The appropriate size and active transport of axonal mitochondria are pivotal for synaptic physiology (25). Defects in axonal mitochondrial dynamics and motility are hallmarks of neuronal mitochondrial pathology in AD, and they reflect mitochondrial functional deficits (24-27). For this reason, we wondered whether OSCP restoration also attenuates the damage to axonal mitochondrial morphology control and mitochondrial trafficking in AD-related conditions. Due to technical difficulty to observe axonal mitochondrial movement in vivo, we employed an in vitro system using cultured neurons from nonTg and Thy- 1 OSCP pups and exposed them to $500 \mathrm{nM}$ oligomeric A $\beta 1-42$ for 24 hours. Scrambled A $\beta 1-42$ at the same concentration was used as a control to determine the specificity of $A \beta$ toxicity. In order to ensure that the in vitro system recapitulates changes that occur in vivo, 
we first performed immunoblotting and observed lowered OSCP expression in oligomeric A $\beta 1$ 42-treated neurons, which was prevented by OSCP overexpression (Supplementary Fig. 3A\&B). In addition to OSCP content, OSCP overexpression rescued mitochondrial bioenergetics from oligomeric $A \beta 1-42$ toxicity as demonstrated by the restoration of mitochondrial membrane potential $(\mathrm{m} \Delta \Psi)($ Supplementary Fig. 4A1\&2) and ATP content (Supplementary Fig. 4B). Furthermore, compared with their nonTg counterparts, oligomeric A $\beta 1-42$-treated OSCPoverexpressing neurons had decreased mitochondrial MitoSox red intensity (Supplementary Fig. 4C1\&2) along with reduced intra-neuronal (Supplementary Fig. 4D1\&3) and intramitochondrial (Supplementary Fig. 4D2\&3) 4-Hydroxynonenal (4-HNE) intensity. These results validate a link between $\mathrm{OSCP}$, mitochondrial defects, and neuronal oxidation due to compromised mitochondrial OXPHOS efficacy in A $\beta$-rich milieus, and they also indicate that our in vitro system reliably replicates changes that occur in vivo. Next, we used mitoDsRed (26) and measured the length of axonal mitochondria to assess axonal mitochondrial dynamics in cultured neurons. OSCP-overexpressing neurons had an average axonal mitochondrial length of $1.62 \pm 0.05 \mu \mathrm{m}$, which was comparable with that of vehicle-treated nonTg neurons $(1.51 \pm 0.06$ $\mu \mathrm{m}, \mathrm{P}>0.05$ ) (Fig. 3A1\&2). Furthermore, there was no significant difference in the distribution patterns of axonal mitochondrial length between the two types of neurons (Fig. 3B). The result indicates that OSCP overexpression does not affect baseline axonal mitochondrial length. Oligomeric A $\beta 1-42$ treatment on nonTg neurons induced a significant reduction in the average axonal mitochondrial length (Fig. 3A1\&2), demonstrated by a leftward shift in the cumulative length distribution (Fig. 3B), suggesting increased axonal mitochondrial fragmentation due to oligomeric A $\beta 1-42$ toxicity. Scrambled A $\beta 1-42$ had no effect on mitochondrial length, indicating the specificity of $A \beta$ toxicity (Fig. 3A\&B). In contrast, OSCP-overexpressing neurons showed 
increased resistance to oligomeric $A \beta 1-42$-induced axonal mitochondrial fission (Fig. 3A\&B). Because two-dimensional measurements of mitochondrial length may not accurately reflect mitochondrial size we also examined axonal mitochondrial volume. Similar to the measures of axonal mitochondrial length, the oligomeric A $\beta 1-42$ application decreased axonal mitochondrial volume, which was mitigated by OSCP overexpression (Fig. 3C1\&2, D). Mitochondria from 910-month-old 5xFAD mice also exhibited elevated phosphorylation of dynamin-like protein 1 (Dlp1) at Ser16 with decreased expression of optic atrophy 1 (Opa1) short (Opa1-S) and long (Opa1-L) isoforms when compared with cells from nonTg controls (Fig. 3E1\&2), further suggesting increased mitochondrial fission in oligomeric A $\beta 1-42$-exposed neurons. These 5xFAD genotypic changes in mitochondrial fusion and fission proteins were rescued by OSCP overexpression (Fig. 3E1\&2). Therefore, we postulate a beneficial effect on mitochondrial dynamics by OSCP overexpression in 5xFAD mice as observed in vitro. Next, we examined axonal mitochondrial trafficking. Oligomeric A $\beta 1-42$-treated neurons had decreased relative numbers of movable mitochondria (Fig. 3G\&J) and increased relative numbers of stationary mitochondria (Fig. 3F\&J) along with reduced anterograde and retrograde movement of mitochondria (Fig. 3H, I, J). These effects of A $\beta$ toxicity on axonal mitochondrial motility correlate with our previous observations (26). Vehicle-treated nonTg and OSCP-overexpressing neurons showed no difference in measures of mitochondrial trafficking (Fig. 3F-J), but OSCP upregulation attenuated oligomeric $A \beta 1-42$-mediated changes in axonal mitochondrial transport (Fig. 3F-J). These findings support a relationship between OSCP deregulation and impaired axonal mitochondrial morphology and movement control in A $\beta$-rich milieus.

\section{Synaptic plasticity is protected in Thy-1 OSCP/5xFAD mice.}


In order to determine whether preservation of neuronal mitochondrial function through OSCP modulation reduces synaptic injury in $5 \mathrm{xFAD}$ mice, we examined synaptic density in the hippocampal CA1 region, which is an AD-sensitive brain area (28), by immunostaining for the vesicular glutamate transporter 1 (vGlut1, to label presynaptic elements) and post synaptic density 95 (PSD95, to label postsynaptic densities) (12). NonTg and Thy-1 OSCP mice showed no differences in their synaptic density at 4-5 and 9-10 months (Fig. 4A1\&2). In contrast, 5xFAD mice showed a large reduction in synaptic elements, which was ameliorated by OSCP overexpression (Fig. 4A1\&2). To determine the functional effects of OSCP modulation on synaptic transmission, we next examined long-term potentiation (LTP) in the hippocampal CA3CA1 pathway (12). Hippocampal slices from 4-5 (Fig. 4B1) and 9-10 (Fig. 4B2) month-old 5xFAD mice showed large deficits in theta burst-evoked LTP as measured by the changes in the slope of the field excitatory postsynaptic potential (fEPSP). The decrease in LTP in 5xFAD mice was lessened through OSCP overexpression (Fig. 4B1\&2) without affecting baseline inputoutput relationships of the evoked responses (Supplementary Fig. 5A\&B). OSCP overexpression did not affect LTP induction or maintenance in non-A $\beta$-expressing mice (Fig. 4A\&B, Supplementary Fig. 5A\&B), indicating that OSCP modulation by itself did not affect synaptic physiology. These findings support a role of OSCP dysfunction in synaptic deficits in 5xFAD mice.

\section{Synapse elimination by microglia is reduced in Thy-1 OSCP/5xFAD mice.}

Recent studies have highlighted synapse elimination through microglial engulfment in $\mathrm{AD}$ $(29,30)$ and they have implicated mitochondrial mechanism of synapse pruning $(31,32)$. In view of the protective effect of OSCP modulation on neuronal mitochondrial function in 5xFAD mice, it is possible that OSCP overexpression may suppress synaptic pruning, contributing to preserved 
synaptic function as seen in Fig. $\mathbf{A A \& B}$. Weak synapses are tagged by the complement system to facilitate microglial engulfment (33) in diseases, including AD (30). To examine whether OSCP overexpression alleviates synapse pruning by microglia in $5 \mathrm{xFAD}$ mice, we first examined complement component 1q (C1q) tagging of synapses in the hippocampal CA1 region in four groups of mice at 4-5 and 9-10 months old through immunolabeling of $\mathrm{C} 1 \mathrm{q}$ and the presynaptic marker synaptophysin. C1q deposition onto synaptophysin-positive synapses was increased in 5xFAD mice as compared with their age- and gender-matched nonTg littermates (Fig. 5A1\&2). In Thy-1 OSCP/5xFAD mice these 5xFAD genotypic effects were attenuated (Fig. 5A1\&2). Consistent with these immunostaining results we also observed augmented C1q content in purified synaptosomes from 9-10-month-old 5xFAD mice by immunoblotting (Supplementary Fig. 6A1\&2), and these alterations were significantly attenuated in Thy-1 OSCP/5xFAD mice (Supplementary Fig. 6A1\&2). C1q tagging of synapses leads to microglial elimination of the tagged synapses (30). Co-staining of synaptophysin and the microglial marker ionized calcium binding adaptor molecule 1 (Iba1) showed increased synaptophysin content in 5xFAD microglia as compared with nonTg mice (Fig. 5B1\&2). OSCP overexpression attenuated these changes in 5xFAD mice (Fig. 5B1\&2). Therefore, the above results indicate enhanced microglial engulfment of synapses in AD-related conditions and a protective effect of OSCP overexpression, which may also contribute to preserved synapse density and strength in OSCPoverexpressing 5xFAD mice.

\section{Spatial learning and memory are improved in Thy-1 OSCP/5xFAD mice.}

Because spatial learning and memory are impaired in AD (34) we examined the influence of OSCP modulation on cognitive functions in the four age- and gender-matched groups of mice at 4-5 and 9-10 months. Consistent with previous studies $(12,13,19), 5 \mathrm{xFAD}$ mice showed 
compromised spatial navigation (Fig. 6A1\&B1) and impaired spatial reference memory (Fig. 6A2\&B2). Consistent with the idea that OSCP overexpression protected synaptic strength, Thy-1 OSCP/5xFAD showed improved spatial navigation (Fig. 6A1\&B1) and spatial reference memory compared with $5 x$ FAD mice (Fig. 6A2\&B2), indicating a protective effect of OSCP modulation on AD-like cognitive impairments.

\section{DISCUSSION}

Amyloid beta $(A \beta)$ is a well-documented key mediator of synaptic injury and cognitive impairment in Alzheimer's disease (AD) (35); however, approaches targeting A $\beta$ have limited disease-modifying effects $(3,4)$. Moreover, the clinical failures of anti-amyloid agents indicate a more complex pathophysiology of $\mathrm{AD}$, which is not likely to be improved through a single therapeutic approach. The mitochondrial cascade hypothesis is based on observations of mitochondrial defects in patients with $\mathrm{AD}$ at both brain and systems levels (5), but it is not a repudiation of $A \beta$ 's importance in the development of $\mathrm{AD}$. Current findings seem to suggest that $\mathrm{A} \beta$ accumulation presents a prelude to mitochondrial deficits, while the latter exaggerates $\mathrm{A} \beta$ neurotoxicity. Consistent with the mitochondrial hypothesis, we previously found a loss of OSCP and $\mathrm{OSCP} / \mathrm{A} \beta$ interaction in $\mathrm{AD}$ brains and 5xFAD mice $(12,13)$. These changes compromise OSCP's role in maintaining F1Fo complex stability and function in neurons (12). Because F1Fo ATP synthase serves an essential function in mitochondrial fitness $(36,37)$, we hypothesized that OSCP contributes to mitochondrial dysfunction and synaptic injury in AD. To test this idea, we targeted OSCP defects and found that OSCP overexpression preserved F1Fo ATP synthase function, preserving mitochondrial function, and protecting axonal mitochondrial dynamics and motility, resulting in improved synaptic strength and cognition. These findings, together with other studies, support the mitochondrial cascade hypothesis of AD and establish a link between 
mitochondrial dysfunction and synaptic deficits. Furthermore, the protective effect of OSCP modulation provides a therapeutic avenue against OSCP dysfunction, which could be a candidate target for future gene therapy.

A critical finding of this study is that OSCP overexpression not only restored OSCP expression, but also attenuated the interaction of $\mathrm{A} \beta$ with mitochondrial membrane-bound OSCP. The occurrence of augmented A $/$ /OSCP complexation in OSCP-overexpressing 5xFAD mitochondria and reduced $\mathrm{A} \beta$ binding to mitochondrial membrane-bond OSCP could be interpreted as protection of functional OSCP that integrates into F1Fo ATP synthase. F1Fo ATP synthase is located on the inner mitochondrial membrane (IMM) (37). The fact that OSCP overexpression had little impact on the expression of other F1Fo ATP synthase subunits suggests that OSCP modulation is not likely to change the components of this enzyme. This suggests that there is abundant "free" OSCP (i.e. non-F1Fo complex-integrated OSCP) in the mitochondrial matrix, which could neutralize $A \beta$ and leave functional OSCP intact. Another possibility is that $\mathrm{A} \beta$-bound OSCP in the F1Fo complex could be replaced by OSCP that is not conjugated to A $\beta$, as the interaction with $\mathrm{A} \beta$ alters OSCP structural conformation and suppresses the connection of OSCP with other subunits (12). Further detailed biostructural studies will help to answer this question. Nevertheless, preserved F1Fo ATP synthase function and integrity serve as strong evidence of preserved OSCP function.

The essential role of mitochondria in supporting synaptic activity has been well documented. The preserved synaptic function in OSCP-overexpressing 5xFAD mice may result from the restoration of mitochondrial energy production, $\mathrm{Ca}^{2+}$ modulating capacity, axonal mitochondrial morphology and positioning control, and suppressed mitochondrial oxidative stress. Recent studies have highlighted microglia-mediated synapse elimination in the 
development of synaptic failure in $\mathrm{AD}$ (30). Although such a process has been largely attributed to microglial activation in response to $A \beta$ toxicity and resultant neuronal death, emerging evidence suggests a mitochondrial role in synapse pruning and the subsequent synaptoapoptosis $(31,32,38)$. We found that $\mathrm{C} 1 \mathrm{q}$ deposition onto synapses was drastically reduced in Thy-1 $\mathrm{OSCP} / 5 \mathrm{xFAD}$ mice as compared to $5 \mathrm{xFAD}$ mice, indicating inhibited complement-dependent synaptic pruning via microglial engulfment in Thy- 1 OSCP/5xFAD mice. Because OSCP modulation preserved mitochondrial function in neurons, we thus propose that mitochondrial defects have multiple effects on the development of synaptic deficits in AD-related conditions.

An important factor that influences these changes is the loss of OSCP in AD-like conditions. We have seen reduced OSCP expression in neurons in AD brains, as well as in A $\beta$ enriched environments such as tissue from 5xFAD mice and oligomeric $A \beta$-exposed neurons $(12,13)$. The association between OSCP downregulation and $\mathrm{A} \beta$ toxicity implicates a relevance of OSCP to AD. We have previously shown an age-dependent effect of OSCP deficiency (11). Advancing age is a major risk factor for AD, especially its sporadic form (39). Indeed, brain aging and $\mathrm{AD}$ exhibit commonalities in several aspects such as DNA injury and alterations in histone modifications, brain oxidative stress and $\mathrm{Ca}^{2+}$ deregulation, as well as synapse loss and microglia-mediated neuroinflammation (40-43). However, AD differs from brain aging in the patterns of structural brain changes and cognitive impairment, as well as the severity of brain pathologies, especially the presence of characteristic $\mathrm{AD}$ lesions including $\mathrm{A} \beta$ plaques and Tauopathy (40-43). In this regard, the relationship of brain aging and AD may be reconciled by a model in which the two pathological states share some mechanistic links at the cellular level, and where the age-dependent development of these changes may serve as critical cofactor and/or promoter for $\mathrm{A} \beta$ toxicity, reinforcing $\mathrm{AD}$ phenotypes. This model underscores the importance of 
exploring age-related factors for the understanding of $\mathrm{AD}$ pathogenesis. Mitochondria play an essential role in synaptic physiology and neuronal survival (44). The strong correlation of brain energy deficiency, oxidative stress, and $\mathrm{Ca}^{2+}$ dysmetabolism with synaptic deficits in both brain aging and $\mathrm{AD}$ supports the hypothesis that mitochondrial dysfunction is a common pathway of synaptic failure in the two disease conditions $(5,45,46)$. Thus, it is possible that OSCP loss is part of a mitochondrial pathway that contributes to the conversion of cognitive aging to dementia, especially in the sporadic form of $\mathrm{AD}$. The deleterious effect of $\mathrm{A} \beta$ on OSCP expression also suggests expedited OSCP loss in AD-related conditions, which may contribute to the rapid deterioration of cognitive functions in prodromal AD. In this regard, modulation of OSCP holds promise to modify cognitive aging for the prevention of $\mathrm{AD}$ onset.

Previous studies have already identified a variety of mitochondrial deficits accompanying $\mathrm{AD}$, and thus the specific contribution of OSCP needs to be further clarified. Mitochondrial defects include dampened mitochondrial bioenergetics, deregulated mitochondrial dynamics and motility, increased permeability of the mitochondrial membrane, enhanced oxidative stress, as well as altered mtDNA regulation (47-50). It is possible that OSCP modulation protects mitochondria through indirect mechanisms. Several mitochondrial proteins, such as amyloid-binding alcohol dehydrogenase (ABAD) (51), cyclophilin D (CypD) (52), and F1Fo ATP synthase $\alpha$ subunit (53) are targets for A $\beta$-binding. OSCP overexpression buffers mitochondrial $A \beta$, which could also reduce the interaction of $A \beta$ with other $A \beta$-binding proteins, providing protection against $A \beta$ toxicity to multiple mitochondrial functions.

\section{CONCLUSIONS}


In summary, we have demonstrated protective effects of OSCP overexpression on $\mathrm{A} \beta$ induced mitochondrial defects and synaptic injury in an in vivo setting. However, several questions remain unanswered, such as the mechanistic link that connects OSCP-associated neuronal mitochondrial dysfunction and microglial phagocytosis of synapses, the mechanisms of OSCP-related axonal mitochondrial morphology and transport control, and whether OSCP loss primes the conversion of cognitive aging to dementia. The simplest interpretation of the available data suggests that OSCP restoration is protective for mitochondrial and synaptic deficits in ADrelevant pathological settings, making OSCP a potential target for the treatment of AD.

\section{ABBREVIATIONS}

OSCP: oligomycin-sensitivity conferring protein

AD: Alzheimer's disease

A $\beta$ : amyloid beta

sA $\beta$ : scramble amyloid beta

OXPHOS: oxidative phosphorylation

ETC: electron transport chain

IMM: inner mitochondrial membrane

nonTg: non-transgenic

Opa1: optic atrophy 1

Dlp1: dynamin-like protein 1 
vGlut1: vesicular glutamate transporter 1

PSD95: post synaptic density 95

LTP: long-term potentiation

fEPSP: field excitatory postsynaptic potential

4-HNE: 4-hydroxynonenal

Co-IP: co-immunoprecipitation

PFA: paraformaldehyde

LFP: local field potential

DAPI: $4^{\prime}$,6-diamidino-2-phenylindole

\section{ETHICS DECLARATIONS}

\section{Ethics approval}

All animals breeding and treatment were approved by Institutional Animal Care and Use Committee (IACUC) at the University of Texas at Dallas (UTD).

\section{CONSENT FOR PUBLICATION}

Not applicable.

\section{AVAILABILITY OF SUPPORTING DATA}

The datasets used and/or analyzed during the current study are available from the corresponding author on reasonable request. 


\section{COMPETING INTERESTS}

The authors have no conflict of interest to claim.

\section{FUNDING}

This work was supported by research funding from NIH (R00AG037716, R01AG053588, and R01AG059753), and Alzheimer's Association (AARG-16-442863).

\section{ACKNOWLEDGEMENT}

Not applicable.

\section{AUTHORS’ INFORMATION}

Esha Gauba and Shaomei Sui contributed equally to this work.

\section{Affiliations}

Department of Biological Sciences, University of Texas at Dallas. Richardson, TX, 75080

Esha Gauba, Shaomei Sui, Jing Tian, Chunxiao Yu, Kun Jia, Tripta Rughwani, Qi Wang, Lan Guo, Heng Du

School of Behavioral and Brain Sciences, University of Texas at Dallas. Richardson, TX, 75080 Christopher Driskill, Sven Kroener,

Authors' contributions: EG, SS, JT, CD, CY, KJ, TR and QW performed experiments. LG and HD conceived the study. SK, LG and HD contributed to data interpretation and experimental design. HD wrote the manuscript. Tienju Wang helped with figure preparation and proofreading. All authors read and approved the final manuscript. 


\title{
Corresponding author
}

\author{
Correspondence to Lan Guo, Heng Du
}

\section{REFERENCES}

1. Querfurth HW, and LaFerla FM. Alzheimer's disease. N Engl J Med. 2010;362(4):329-44.

2. Hardy JA, and Higgins GA. Alzheimer's disease: the amyloid cascade hypothesis. Science. 1992;256(5054):184-5.

3. Honig LS, Vellas B, Woodward M, Boada M, Bullock R, Borrie M, et al. Trial of Solanezumab for Mild Dementia Due to Alzheimer's Disease. N Engl J Med. 2018;378(4):321-30.

4. Selkoe DJ. Alzheimer disease and aducanumab: adjusting our approach. Nat Rev Neurol. 2019;15(7):365-6.

5. Swerdlow RH. Mitochondria and Mitochondrial Cascades in Alzheimer's Disease. J Alzheimers Dis. 2018;62(3):1403-16.

6. Reddy PH. Amyloid beta, mitochondrial structural and functional dynamics in Alzheimer's disease. Exp Neurol. 2009;218(2):286-92.

7. Mosconi L, Pupi A, and De Leon MJ. Brain glucose hypometabolism and oxidative stress in preclinical Alzheimer's disease. Ann N Y Acad Sci. 2008;1147:180-95.

8. Swerdlow RH, Burns JM, and Khan SM. The Alzheimer's disease mitochondrial cascade hypothesis: progress and perspectives. Biochim Biophys Acta. 2014;1842(8):1219-31.

9. Walker JE, Runswick MJ, and Poulter L. ATP synthase from bovine mitochondria. The characterization and sequence analysis of two membrane-associated sub-units and of the corresponding cDNAs. J Mol Biol. 1987;197(1):89-100.

10. Walker JE. ATP Synthesis by Rotary Catalysis (Nobel lecture). Angew Chem Int Ed Engl. 1998;37(17):2308-19.

11. Gauba E, Guo L, and Du H. Cyclophilin D Promotes Brain Mitochondrial F1FO ATP Synthase Dysfunction in Aging Mice. J Alzheimers Dis. 2017;55(4):1351-62.

12. Beck SJ, Guo L, Phensy A, Tian J, Wang L, Tandon N, et al. Deregulation of mitochondrial F1FOATP synthase via OSCP in Alzheimer's disease. Nat Commun. 2016;7:11483.

13. Gauba E, Chen H, Guo L, and Du H. Cyclophilin D deficiency attenuates mitochondrial F1Fo ATP synthase dysfunction via OSCP in Alzheimer's disease. Neurobiol Dis. 2019;121:138-47.

14. Rubinstein JL, Walker JE, and Henderson R. Structure of the mitochondrial ATP synthase by electron cryomicroscopy. EMBO J. 2003;22(23):6182-92.

15. Wilkens S, and Capaldi RA. ATP synthase's second stalk comes into focus. Nature. 1998;393(6680):29.

16. Du H, Guo L, Yan S, Sosunov AA, McKhann GM, and Yan SS. Early deficits in synaptic mitochondria in an Alzheimer's disease mouse model. Proc Natl Acad Sci U S A. 2010;107(43):18670-5.

17. Guo L, Tian J, and Du H. Mitochondrial Dysfunction and Synaptic Transmission Failure in Alzheimer's Disease. J Alzheimers Dis. 2017;57(4):1071-86.

18. Ly CV, and Verstreken P. Mitochondria at the synapse. Neuroscientist. 2006;12(4):291-9.

19. Wang L, Guo L, Lu L, Sun H, Shao M, Beck SJ, et al. Synaptosomal Mitochondrial Dysfunction in 5xFAD Mouse Model of Alzheimer's Disease. PLoS One. 2016;11(3):e0150441. 
20. Joshi S, Cao GJ, Nath C, and Shah J. Oligomycin sensitivity conferring protein (OSCP) of bovine heart mitochondrial ATP synthase: high-affinity OSCP-Fo interactions require a local alpha-helix at the C-terminal end of the subunit. Biochemistry. 1997;36(36):10936-43.

21. Bonora M, Morganti C, Morciano G, Pedriali G, Lebiedzinska-Arciszewska M, Aquila G, et al. Mitochondrial permeability transition involves dissociation of F1FO ATP synthase dimers and Cring conformation. EMBO Rep. 2017;18(7):1077-89.

22. Amodeo GF, Solesio ME, and Pavlov EV. From ATP synthase dimers to C-ring conformational changes: unified model of the mitochondrial permeability transition pore. Cell Death Dis. 2017;8(12):1.

23. Alavian KN, Beutner G, Lazrove E, Sacchetti S, Park HA, Licznerski P, et al. An uncoupling channel within the c-subunit ring of the F1FO ATP synthase is the mitochondrial permeability transition pore. Proc Natl Acad Sci U S A. 2014;111(29):10580-5.

24. Su B, Wang X, Zheng L, Perry G, Smith MA, and Zhu X. Abnormal mitochondrial dynamics and neurodegenerative diseases. Biochim Biophys Acta. 2010;1802(1):135-42.

25. Sheng $\mathrm{ZH}$, and Cai Q. Mitochondrial transport in neurons: impact on synaptic homeostasis and neurodegeneration. Nat Rev Neurosci. 2012;13(2):77-93.

26. Guo L, Du H, Yan S, Wu X, McKhann GM, Chen JX, et al. Cyclophilin D deficiency rescues axonal mitochondrial transport in Alzheimer's neurons. PLoS One. 2013;8(1):e54914.

27. Manczak M, Calkins MJ, and Reddy PH. Impaired mitochondrial dynamics and abnormal interaction of amyloid beta with mitochondrial protein Drp1 in neurons from patients with Alzheimer's disease: implications for neuronal damage. Hum Mol Genet. 2011;20(13):2495-509.

28. Padurariu M, Ciobica A, Mavroudis I, Fotiou D, and Baloyannis S. Hippocampal neuronal loss in the CA1 and CA3 areas of Alzheimer's disease patients. Psychiatr Danub. 2012;24(2):152-8.

29. Rajendran L, and Paolicelli RC. Microglia-Mediated Synapse Loss in Alzheimer's Disease. J Neurosci. 2018;38(12):2911-9.

30. Hong S, Beja-Glasser VF, Nfonoyim BM, Frouin A, Li S, Ramakrishnan S, et al. Complement and microglia mediate early synapse loss in Alzheimer mouse models. Science. 2016;352(6286):7126.

31. Meng L, Mulcahy B, Cook SJ, Neubauer M, Wan A, Jin Y, et al. The Cell Death Pathway Regulates Synapse Elimination through Cleavage of Gelsolin in Caenorhabditis elegans Neurons. Cell Rep. 2015;11(11):1737-48.

32. Gyorffy BA, Kun J, Torok G, Bulyaki E, Borhegyi Z, Gulyassy P, et al. Local apoptotic-like mechanisms underlie complement-mediated synaptic pruning. Proc Natl Acad Sci U S A. 2018;115(24):6303-8.

33. Presumey J, Bialas AR, and Carroll MC. Complement System in Neural Synapse Elimination in Development and Disease. Adv Immunol. 2017;135:53-79.

34. Adelstein TB, Kesner RP, and Strassberg DS. Spatial recognition and spatial order memory in patients with dementia of the Alzheimer's type. Neuropsychologia. 1992;30(1):59-67.

35. Selkoe DJ. Alzheimer's disease is a synaptic failure. Science. 2002;298(5594):789-91.

36. Walker JE. The ATP synthase: the understood, the uncertain and the unknown. Biochem Soc Trans. 2013;41(1):1-16.

37. Trombetti F, Pagliarani A, Ventrella V, Algieri C, and Nesci S. Crucial aminoacids in the FO sector of the F1FO-ATP synthase address $\mathrm{H}(+)$ across the inner mitochondrial membrane: molecular implications in mitochondrial dysfunctions. Amino Acids. 2019;51(4):579-87.

38. Sidlauskaite E, Gibson JW, Megson IL, Whitfield PD, Tovmasyan A, Batinic-Haberle I, et al. Mitochondrial ROS cause motor deficits induced by synaptic inactivity: Implications for synapse pruning. Redox Biol. 2018;16:344-51.

39. Guerreiro R, and Bras J. The age factor in Alzheimer's disease. Genome Med. 2015;7:106. 
40. Denver P, and McClean PL. Distinguishing normal brain aging from the development of Alzheimer's disease: inflammation, insulin signaling and cognition. Neural Regen Res. 2018;13(10):1719-30.

41. Swerdlow RH. Brain aging, Alzheimer's disease, and mitochondria. Biochim Biophys Acta. 2011;1812(12):1630-9.

42. Hof PR, and Morrison JH. The aging brain: morphomolecular senescence of cortical circuits. Trends Neurosci. 2004;27(10):607-13.

43. Zhang HY, Chen WX, Jiao $Y, X u Y$, Zhang XR, and Wu JT. Selective vulnerability related to aging in large-scale resting brain networks. PLoS One. 2014;9(10):e108807.

44. Lee A, Hirabayashi Y, Kwon SK, Lewis TL, Jr., and Polleux F. Emerging roles of mitochondria in synaptic transmission and neurodegeneration. Curr Opin Physiol. 2018;3:82-93.

45. Muller WE, Eckert A, Kurz C, Eckert GP, and Leuner K. Mitochondrial dysfunction: common final pathway in brain aging and Alzheimer's disease--therapeutic aspects. Mol Neurobiol. 2010;41(23):159-71.

46. Reddy PH, and Beal MF. Amyloid beta, mitochondrial dysfunction and synaptic damage: implications for cognitive decline in aging and Alzheimer's disease. Trends $\mathrm{Mol}$ Med. 2008;14(2):45-53.

47. Wilkins HM, Koppel SJ, Weidling IW, Roy N, Ryan LN, Stanford JA, et al. Extracellular Mitochondria and Mitochondrial Components Act as Damage-Associated Molecular Pattern Molecules in the Mouse Brain. J Neuroimmune Pharmacol. 2016;11(4):622-8.

48. Wang X, Su B, Lee HG, Li X, Perry G, Smith MA, et al. Impaired balance of mitochondrial fission and fusion in Alzheimer's disease. J Neurosci. 2009;29(28):9090-103.

49. Manczak M, Anekonda TS, Henson E, Park BS, Quinn J, and Reddy PH. Mitochondria are a direct site of $A$ beta accumulation in Alzheimer's disease neurons: implications for free radical generation and oxidative damage in disease progression. Hum Mol Genet. 2006;15(9):1437-49.

50. Ye X, Sun X, Starovoytov V, and Cai Q. Parkin-mediated mitophagy in mutant hAPP neurons and Alzheimer's disease patient brains. Hum Mol Genet. 2015;24(10):2938-51.

51. Lustbader JW, Cirilli M, Lin C, Xu HW, Takuma K, Wang N, et al. ABAD directly links Abeta to mitochondrial toxicity in Alzheimer's disease. Science. 2004;304(5669):448-52.

52. Du H, Guo L, Fang F, Chen D, Sosunov AA, McKhann GM, et al. Cyclophilin D deficiency attenuates mitochondrial and neuronal perturbation and ameliorates learning and memory in Alzheimer's disease. Nat Med. 2008;14(10):1097-105.

53. Schmidt C, Lepsverdize E, Chi SL, Das AM, Pizzo SV, Dityatev A, et al. Amyloid precursor protein and amyloid beta-peptide bind to ATP synthase and regulate its activity at the surface of neural cells. Mol Psychiatry. 2008;13(10):953-69.

54. Feng G, Mellor RH, Bernstein M, Keller-Peck C, Nguyen QT, Wallace M, et al. Imaging neuronal subsets in transgenic mice expressing multiple spectral variants of GFP. Neuron. 2000;28(1):4151.

55. Salin K, Villasevil EM, Auer SK, Anderson GJ, Selman C, Metcalfe NB, et al. Simultaneous measurement of mitochondrial respiration and ATP production in tissue homogenates and calculation of effective P/O ratios. Physiol Rep. 2016;4(20).

56. Tian J, Guo L, Sui S, Driskill C, Phensy A, Wang Q, et al. Disrupted hippocampal growth hormone secretagogue receptor 1alpha interaction with dopamine receptor D1 plays a role in Alzheimer's disease. Sci Transl Med. 2019;11(505).

57. H D, Guo L, Yan S, Sosunov AA, McKhann GM, and Yan SS. Early deficits in synaptic mitochondria in an Alzheimer's disease mouse model. Proceedings of the National Academy of Sciences of the United States of America. 2010;107(43):18670-5. 
A1 पnonTg $\square$ 5xFAD $\square$ Thy-1 OSCP $\square$ Thy-1 OSCP/5xFAD
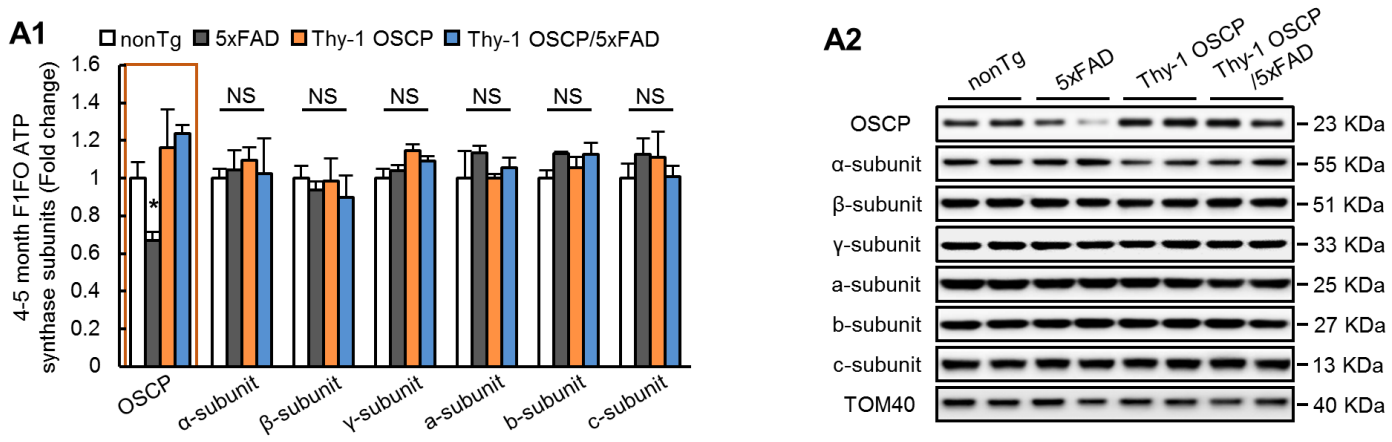

B1 口nonTg $\square$ 5xFAD $\square$ Thy-1 OSCP $\square$ Thy-1 OSCP/5xFAD
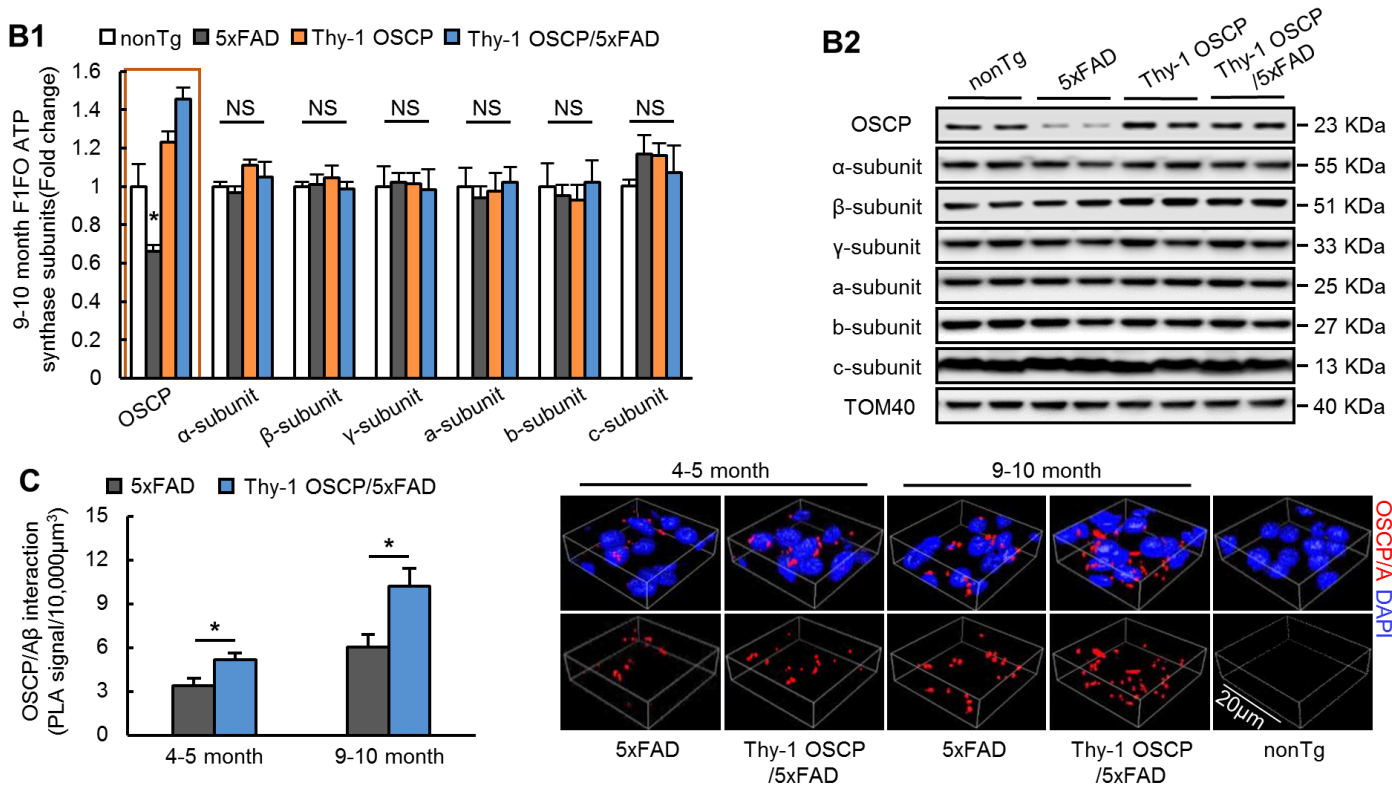

9-10 month
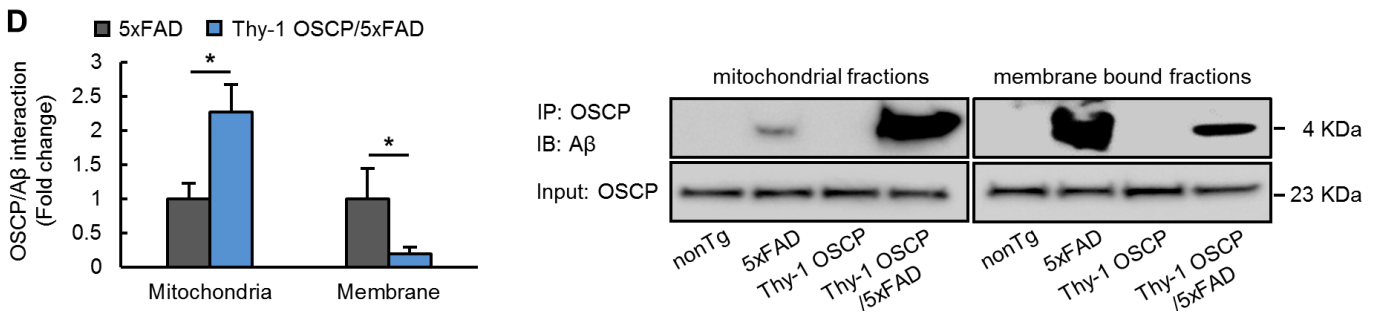

Fig. 1 Alleviated OSCP aberrations in Thy-1 OSCP/5xFAD mice. (A\&B) Immunoblotting analysis of OSCP and other major subunits of F1FO ATP synthase including $\alpha, \beta, \gamma, \mathrm{a}, \mathrm{b}$, and $\mathrm{c}$ in brain mitochondria isolated from 4-5 (A1) and 9-10-month-old (B1) nonTg, 5xFAD, Thy-1 OSCP, and Thy-1 OSCP/5xFAD mice. TOM40 was used as loading control. Two-way ANOVA followed by Bonferroni post hoc analysis. ${ }^{*} P<0.05$ 5xFAD vs other groups; NS, not 
significant. $n=3-7$ mice per group. (A2\&B2) Representative bands of immunoblotting. (C) Duolink PLA positive dots for OSCP/A $\beta$ complex in cortex from 4-5 and 9-10-month-old 5xFAD and Thy-1 OSCP/5xFAD mice. Unpaired student $t$-test. $* P<0.05 . n=4-5$ mice per group. Right panel are the 3-dimensional (3D) representative images of OSCP/A $\beta$ PLA positive dots in cortex. Red dots represent OSCP/A $\beta$ interaction, DAPI labels cell nuclei. Scale bar $=20$ $\mu \mathrm{m}$. (D) Co-immunoprecipitation (Co-IP) of OSCP and A $\beta$ in isolated mitochondria or their membrane fractions from 9-10-month-old nonTg, 5xFAD, Thy-1 OSCP, and Thy-1 OSCP/5xFAD mice. Right panel are the representative images of Co-IP. Unpaired student $t$-test. * $P<0.05 . n=4-5$ mice per group. 


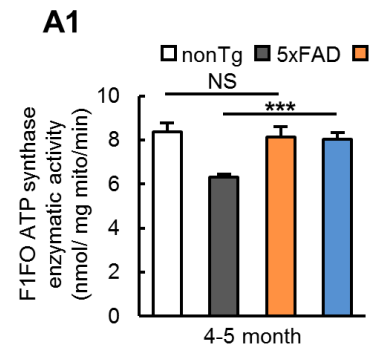

C1

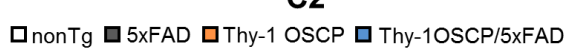

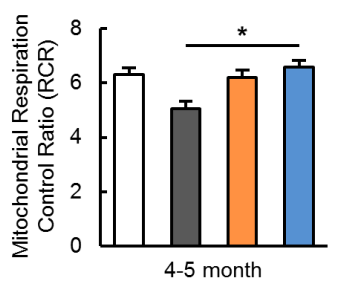

E1

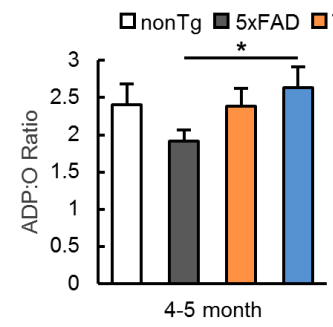

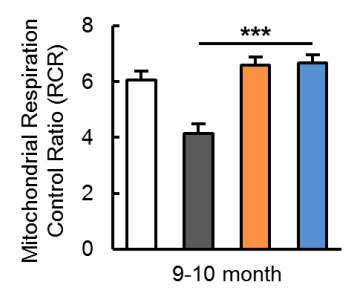

A2

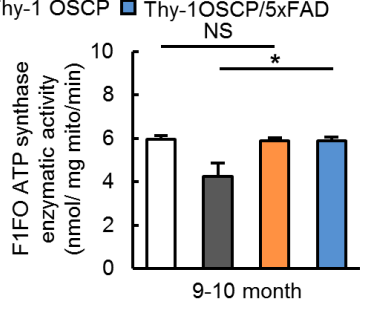

C2

\section{E2}

1 OSCP ם Thy-1OSCP/5xFAD

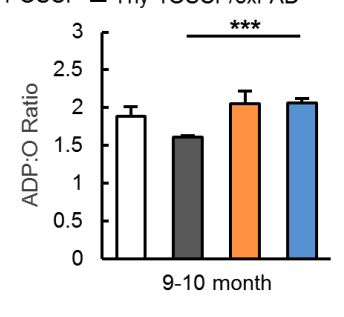

B1

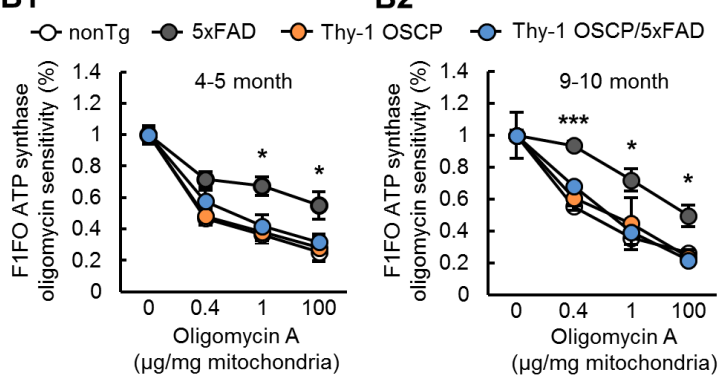

D1

D2
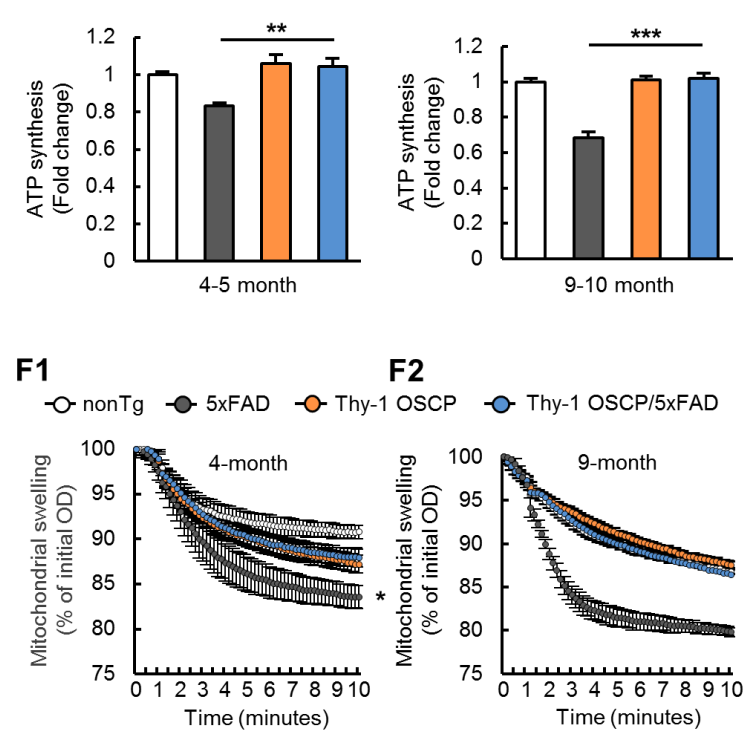

F2

OSCP $-\mathrm{O}$ Thy-1 OSCP/5xFAD

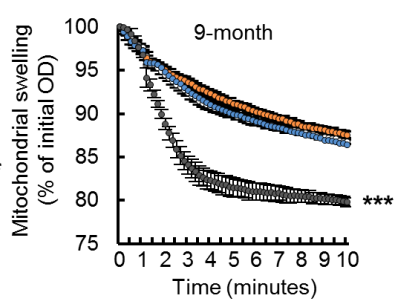

Fig. 2 Attenuated mitochondrial dysfunction in Thy-1 OSCP/5xFAD mice. (A) F1FO ATP synthase catalytic activity of brain mitochondria isolated from 4-5 (A1) and 9-10-month-old (A2) nonTg, 5xFAD, Thy-1 OSCP, and Thy-1 OSCP/5xFAD mice. Unpaired student $t$-test. * $P<$ $0.05, * * * P<0.001$; NS, not significant. $n=4-7$ mice per group. (B) Oligomycin sensitivity of isolated brain mitochondria from 4-5 (B1) and 9-10-month-old (B2) nonTg, 5xFAD, Thy-1 OSCP, and Thy-1 OSCP/5xFAD mice. Unpaired student $t$-test. $* P<0.05$ 5xFAD vs Thy1OSCP/5xFAD, *** $P<0.0015 x F A D$ vs Thy1-OSCP/5xFAD. $n=3-7$ mice per group. (C) Mitochondrial respiration control ratio in 4-5 (C1) and 9-10-month-old (C2) old nonTg, 5xFAD, Thy-1 OSCP, and Thy-1 OSCP/5xFAD mice. Unpaired student $t$-test. $* P<0.05$, $* * * P<0.001$. $n=5-7$ mice per group. (D) ATP production in isolated brain mitochondria from 4-5 (D1) and 910-month (D2) nonTg, 5xFAD, Thy-1 OSCP, and Thy-1 OSCP/5xFAD mice. Unpaired student $t$-test. $* * P<0.01, * * * P<0.001 . n=4-6$ mice per group. (E) Mitochondrial ATP:O ratio in 4-5 (E1) and 9-10-month-old (E2) nonTg, 5xFAD, Thy-1 OSCP and Thy-1 OSCP/5xFAD mice. Unpaired student $t$-test. $* P<0.05$ vs other groups, $* * * P<0.001 . n=4-6$ mice per group. (F) Mitochondrial swelling for 4-5 (F1) and 9-10-month-old (F2) nonTg, 5xFAD, Thy-1 OSCP, and Thy-1 OSCP/5xFAD mice. Unpaired student $t$-test. $* P<0.055 \times$ FAD vs Thy1-OSCP/5xFAD, *** $P<0.0015 x$ FAD vs Thyl-OSCP/5xFAD. $n=5-9$ mice per group. 

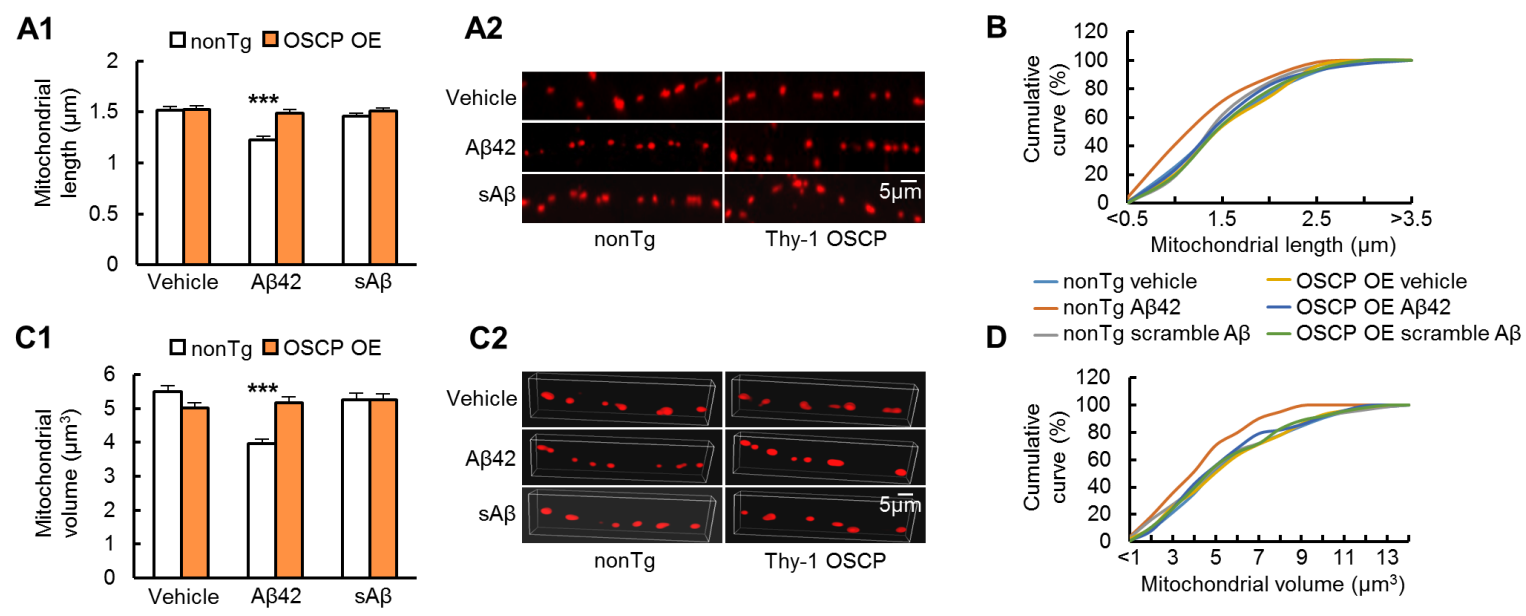

E1
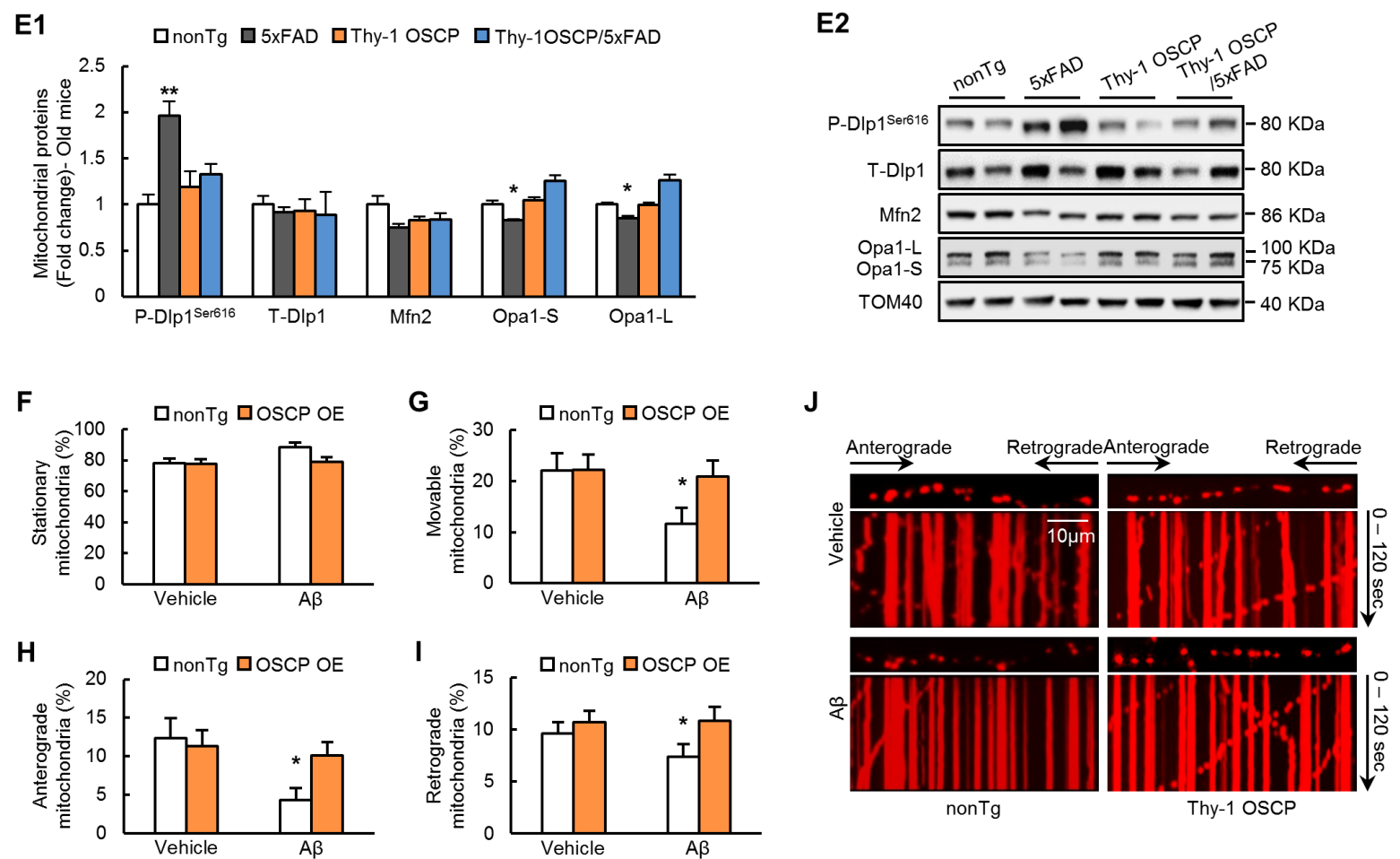

Thy-1 OSCP

Fig. 3 Rescued axonal mitochondrial dynamic and motility by OSCP overexpression in A $\beta$ treated neurons. (A1) The average length of axonal mitochondria was measured in primary nonTg and Thy1-OSCP neurons expressing mitoDsred. The neurons were treated with vehicle, 1 $\mu \mathrm{M} A \beta 1-42$ or $1 \mu \mathrm{M}$ scramble $\mathrm{A} \beta$ for 24 hours. Two-way ANOVA followed by Bonferroni post hoc analysis. ${ }^{* * *} P<0.001 . n=227-256$ mitochondria per group. (A2) Representative images of axonal mitochondria in primary cultured neurons. Scale bar $=5 \mu \mathrm{m}$. (B) Cumulative distribution data of axonal mitochondrial length. $n=227-256$ mitochondria per group. (C1) The average volume of axonal mitochondria was analyzed from nonTg and Thy1-OSCP neurons treated with vehicle, $1 \mu \mathrm{M} \mathrm{A} \beta 1-42$ or $1 \mu \mathrm{M}$ scramble $\mathrm{A} \beta$ for 24 hours. Two-way ANOVA followed by Bonferroni post hoc analysis. $* * * P<0.001 . n=223-256$ mitochondria per group. (C2) Representative 3D images of axonal mitochondria in primary cultured neurons. Scale bar $=5$ $\mu \mathrm{m}$. (D) Cumulative distribution data of axonal mitochondrial volume. $n=223-256$ 
mitochondria per group. (E1) Immunoblotting analysis of mitochondrial dynamic protein in isolated brain mitochondria from 9-10-month-old nonTg, 5xFAD, Thy-1 OSCP, and Thy-1 OSCP/5xFAD mice. p-Dlp1 ${ }^{\text {Ser616 }}$, T-Dlp1, Mfn2, and OPA1 including short form (OPA1-S) and long form (OPA1-L) were detected. Two-way ANOVA followed by Bonferroni post hoc analysis. ${ }^{*} P<0.05$ vs other groups. ${ }^{* *} P<0.01$ vs other groups. $n=3-4$ mice per group. (E2) Representative bands of immunoblotting in E1. (F-J) Axonal mitochondrial trafficking including percentage of stationary axonal mitochondria $(\mathbf{F})$, percentages of movable $(\mathbf{G})$, anterograding $(\mathbf{H})$, and retrograding (I) mitochondria for vehicle and 24 hours $1 \mu \mathrm{M} \mathrm{A} \beta 1-42$ or $1 \mu \mathrm{M}$ scramble A $\beta$-treated nonTg and Thy1-OSCP neurons. Unpaired student $t$-test. ${ }^{*} P<0.05$ vs other groups. (J) Representative kymographs of axonal mitochondrial movement. Scale bar $=10 \mu \mathrm{m}$. Data were collected from 3 independent experiments. 
A1
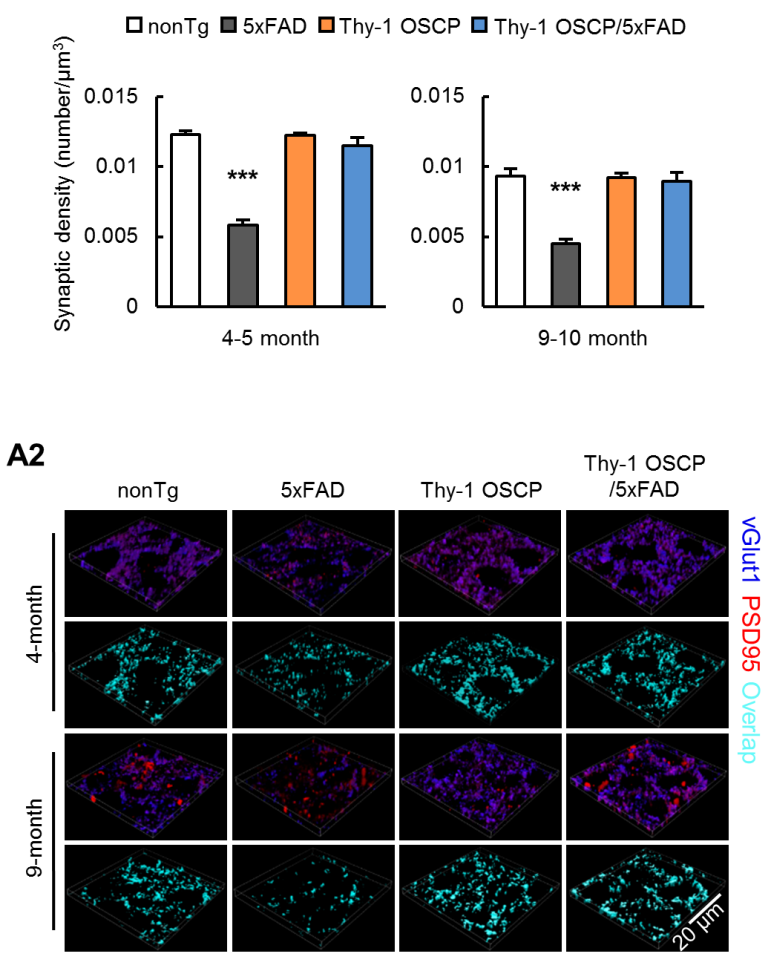

B1
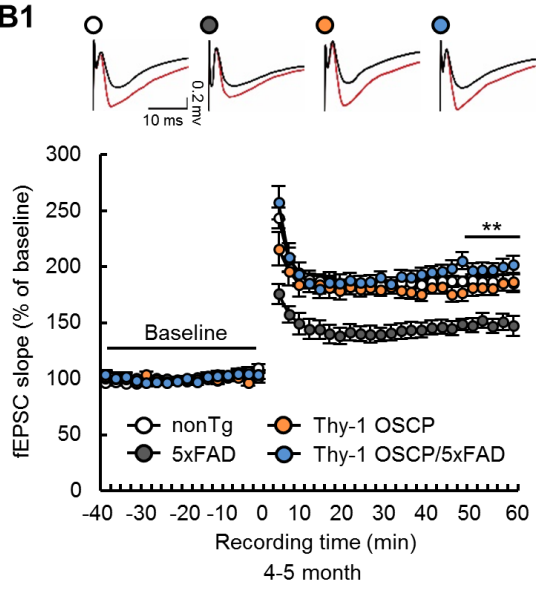

B2
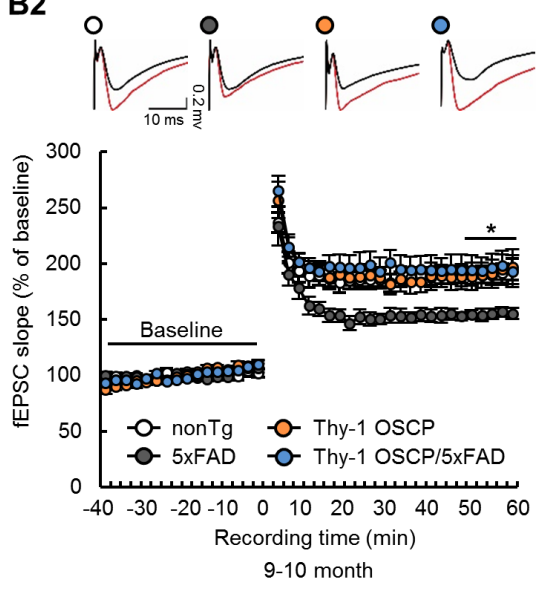

Fig. 4 Preserved synaptic plasticity and transmission in Thy-1 OSCP/5xFAD mice. (A1) Synaptic density of 4-5-and 9-10-month-old nonTg, 5xFAD, Thy-1 OSCP, and Thy-1 OSCP/5xFAD mice. Two-way ANOVA followed by Bonferroni post hoc analysis. *** $P<$ 0.001 vs other groups. $n=5$ mice per group. (A2) Representative 3D-reconstructed images of synapse staining. vGLUT1 (blue) and PSD95 (red) were used to visualize pre- and post-synaptic components, respectively. The overlaid staining of vGLUT1 and PSD95 indicates synapses. Scale bar $=20 \mu \mathrm{m}$. (B) Time course of long-term-potential (LTP) and representative fEPSP responses during the baseline period (black trace) and 30 seconds after theta burst stimulation (red trace) in four groups of mice at 4-5 (B1) and 9-10 (B2) months old. Two-way ANOVA followed by Bonferroni post hoc analysis. ${ }^{*} P<0.055 \mathrm{xFAD}$ vs other groups. $* * P<0.01$ $5 \times$ FAD vs other groups. $n=6-9$ mice per group. 
A1

口nonTg $\square$ 5xFAD $\square$ Thy-1 OSCP $\square$ Thy-1 OSCP/5xFAD
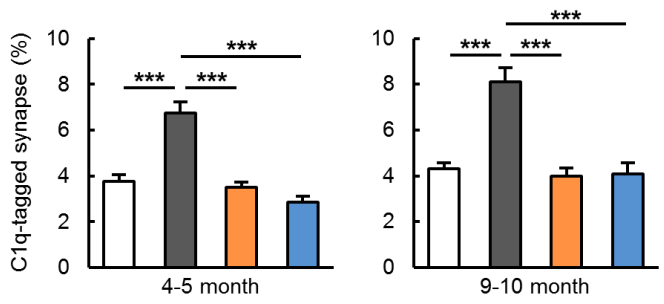

A2

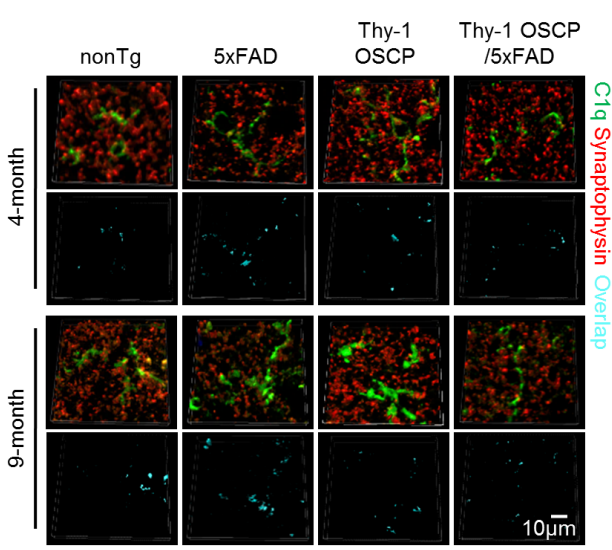

B1

DnonTg $\square$ 5xFAD $\square$ Thy-1 OSCP $\square$ Thy-1 OSCP/5xFAD
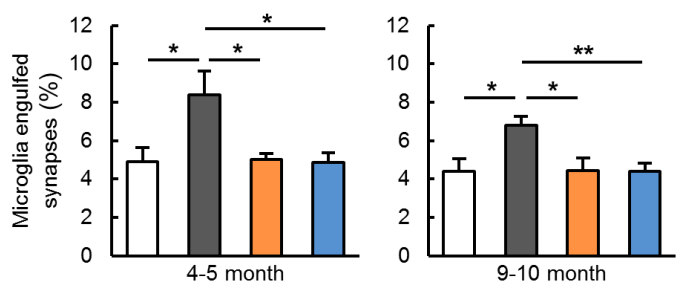

B2

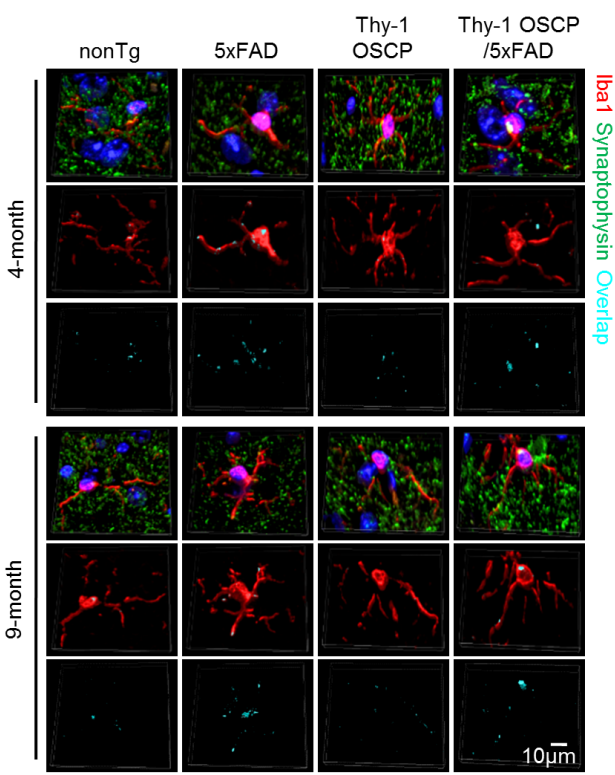

Fig. 5 Mitigated synapse trimming by microglia in Thy-1 OSCP/5xFAD mice. (A1) Analysis of C1q-tagged synapses in cortex from 4-5 and 9-10-month-old nonTg, 5xFAD, Thy-1 OSCP, and Thy-1 OSCP/5xFAD mice. Two-way ANOVA followed by Bonferroni post hoc analysis. *** $P<0.001$. $n=5$ mice per group. (A2) Representative 3D-reconstructed images of C1qtagged synapses in cortex. Synaptophysin (red) represents synapses. The overlaid staining of C1q and synaptophysin indicates C1q-tagged synapses. Scale bar $=10 \mu \mathrm{m}$. (B1) Synaptic pruning was examined through co-staining of microglia (Iba1, red) and synapses (synaptophysin, green). Two-way ANOVA followed by Bonferroni post hoc analysis. ${ }^{*} P<0.05, * * P<0.01 . n=5$ mice per group. (B2) 3D representative images of synaptic pruning by microglia. The overlaid staining of Iba1 and synaptophysin indicates microglia-engulfed synapses. Scale bar $=5 \mu \mathrm{m}$. 


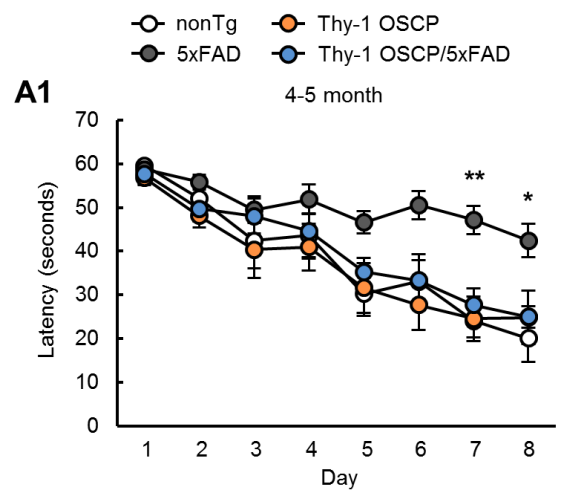

DnonTg $\square$ 5xFAD $\square$ Thy-1 OSCP $\square$ Thy-1 OSCP/5xFAD
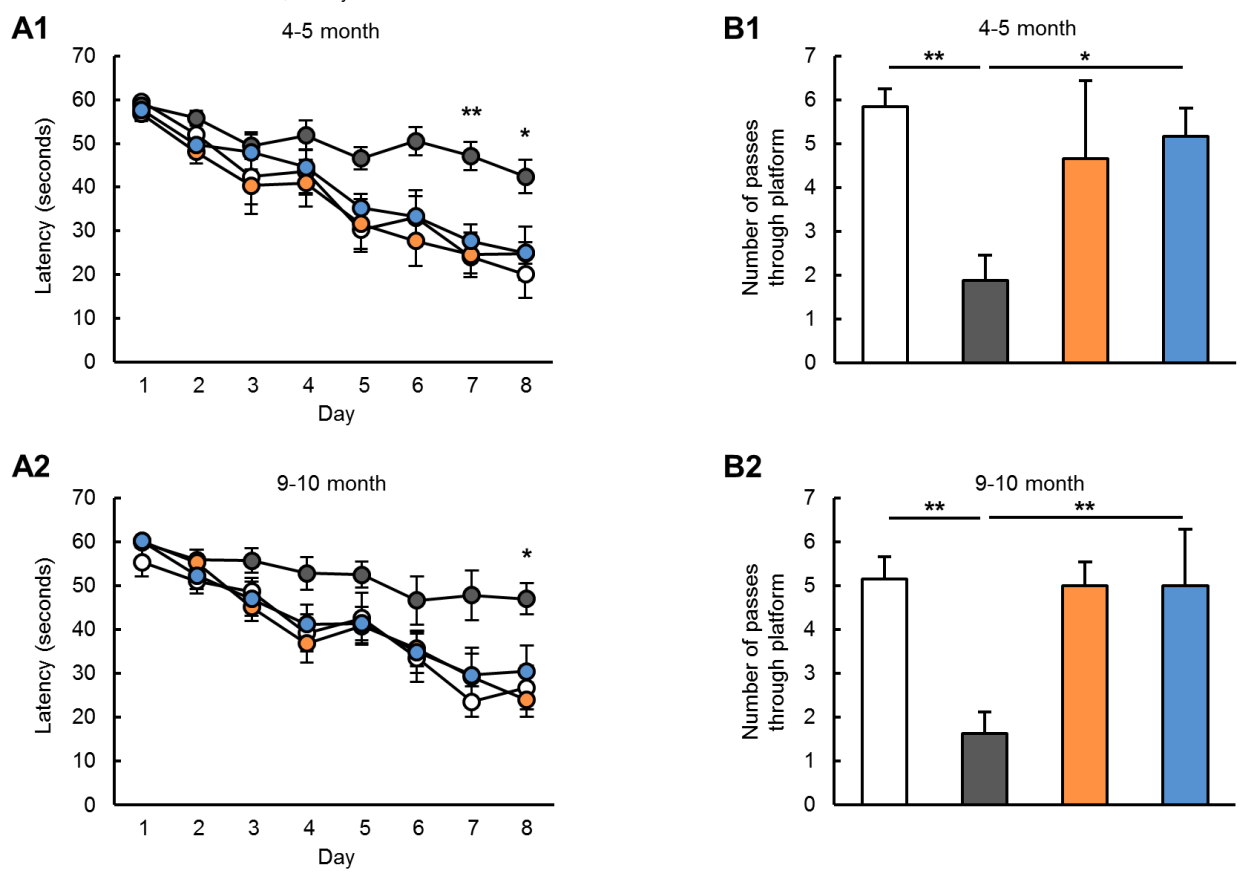

Figure 6. Improved cognitive function in Thy-1 OSCP/5xFAD mice. Spatial learning $(\mathbf{A} 1 \& \mathbf{A 2})$ and reference memory (B1\&B2) of 4-5 and 9-10-month-old nonTg, 5xFAD, Thy-1 OSCP, and Thy-1 OSCP/5xFAD mice. One-way ANOVA followed by Bonferroni post hoc analysis. ${ }^{*} P<0.05$ vs other groups, ${ }^{* *} P<0.01$ vs other groups. $n=6-9$ mice per group. 

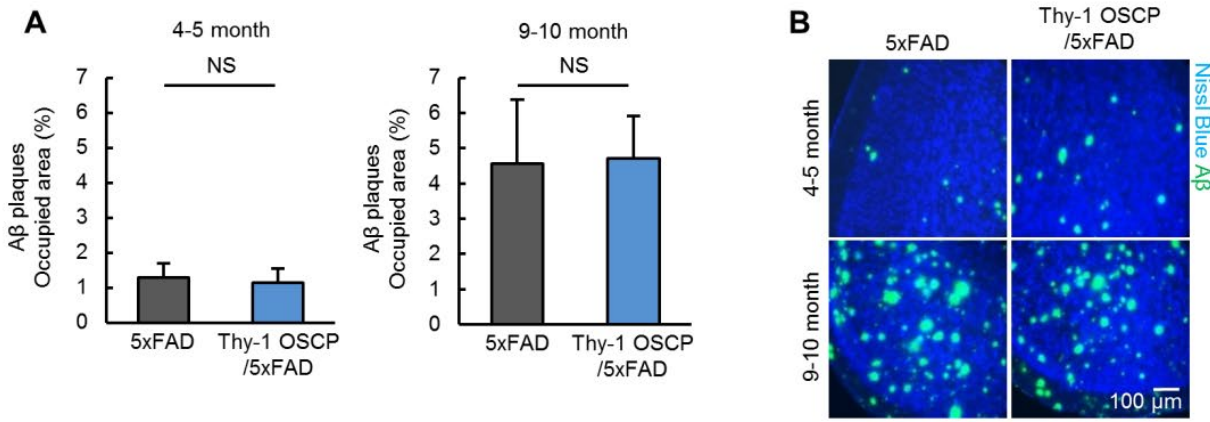

sFig. 1 Unaltered A $\beta$ deposition in cortex in Thy-1 OSCP/5xFAD mice compared to 5xFAD mice. (A) $A \beta$ deposition in the cortex was measured and analyzed by immunostaining using $A \beta$ antibody in 5xFAD and Thy-1 OSCP/5xFAD mice at 4-5 and 9-10 months old. Unpaired student $t$-test, $n=5$-6 mice per group. NS, not significant. (B) Representative images for amyloid plaques in cortex. Scale bar $=100 \mu \mathrm{m}$. 

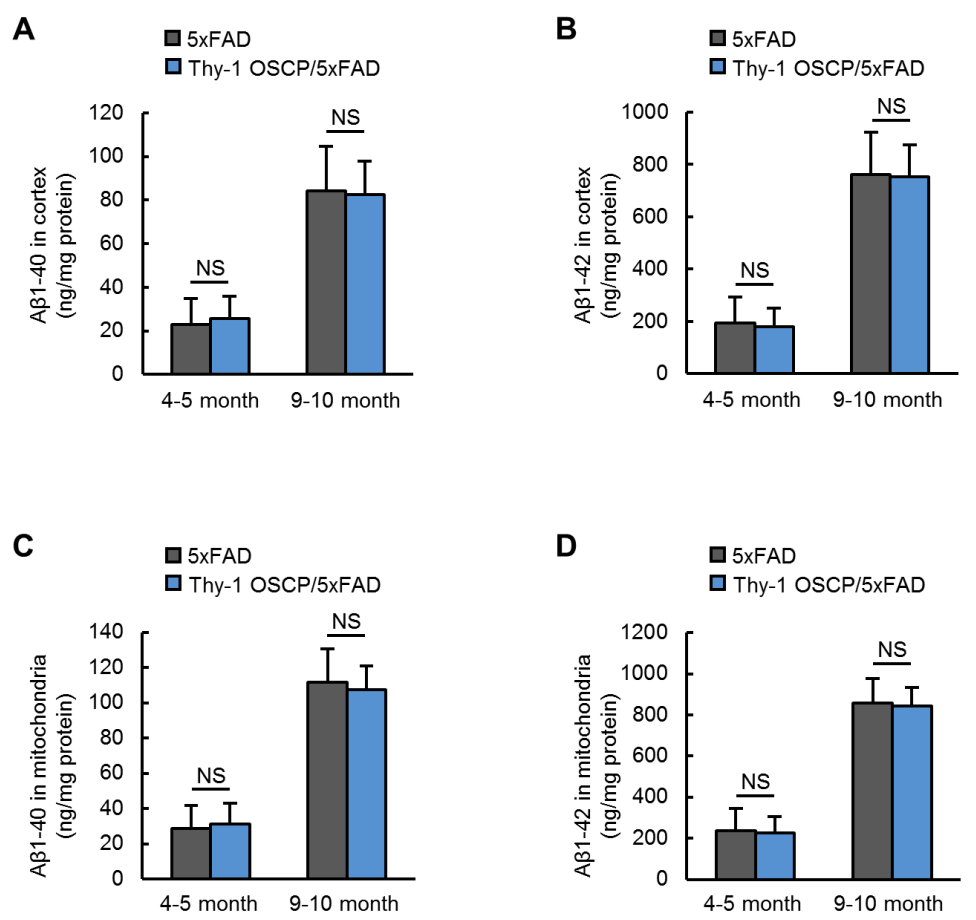

sFig. 2 Unaltered soluble A $\beta 1-40$ and 1-42 amounts in cortex in Thy-1 OSCP/5xFAD mice compared to 5xFAD mice. (A) Cortical soluble A $\beta$ 1-40 and 1-42 were detected using ELISA assay in 5xFAD and Thy-1 OSCP/5xFAD mice at 4-5 and 9-10 months old. Unpaired student $t$ test, 4-5month: 5xFAD, $n=6$, Thy-1 OSCP/5xFAD, $n=6$; 9-10-month: 5xFAD, $n=5$, Thy- 1 $\mathrm{OSCP} / 5 \mathrm{xFAD}, n=6$. NS, not significant. (B) Mitochondrial soluble A $\beta$ 1-40 and 1-42 were detected using ELISA assay for 5xFAD and Thy-1 OSCP/5xFAD mice at 4-5 months and 9-10 months old. Unpaired student $t$-test, 4-5-month: 5xFAD, $n=6$, Thy- 1 OSCP/5xFAD, $n=6$; 910-month: 5xFAD, $n=5$, Thy-1 OSCP/5xFAD, $n=6$. NS, not significant. 
A

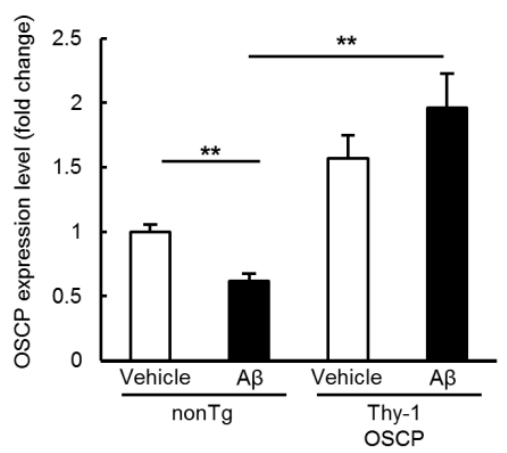

B

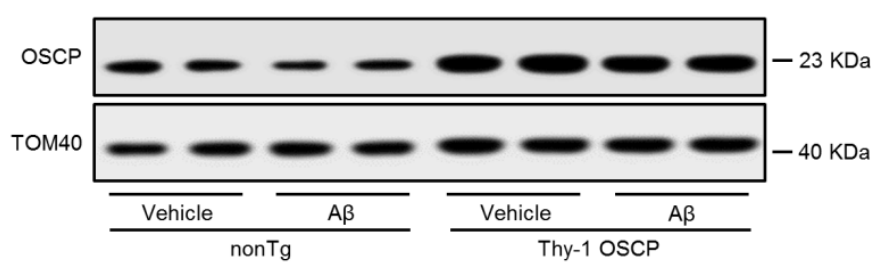

sFig. 3 OSCP expression in Aß1-42 treated neurons. (A) Immunoblotting analysis of OSCP in nonTg and Thy1-OSCP neurons treated with vehicle or $1 \mu \mathrm{M} \mathrm{A} \beta 42$ for 24 hours. TOM40 was used as a loading control. Unpaired student $t$-test. ${ }^{* *} P<0.01 . n=5$. (B) Representative bands of OSCP and TOM40 in primary neurons. 


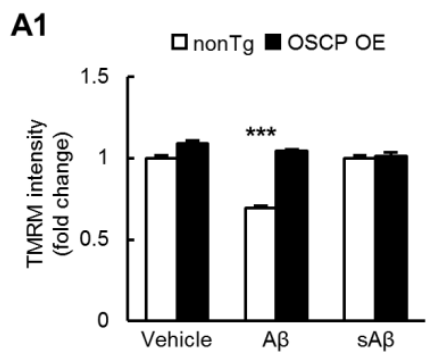

C1 1 nonTg $\mathbf{M S C P}$ OE

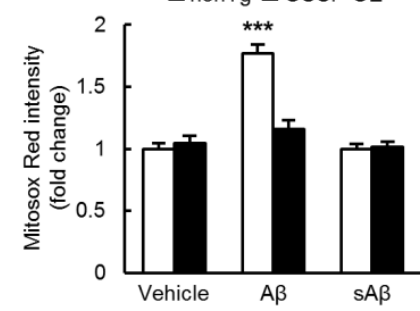

D1

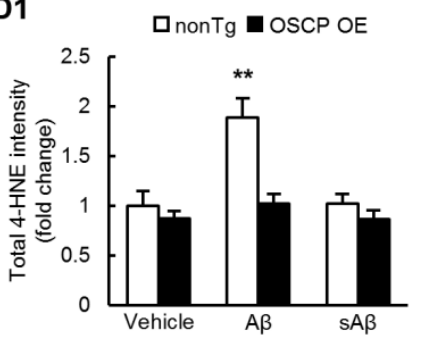

D2

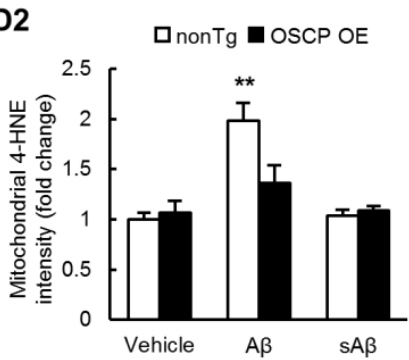

A2

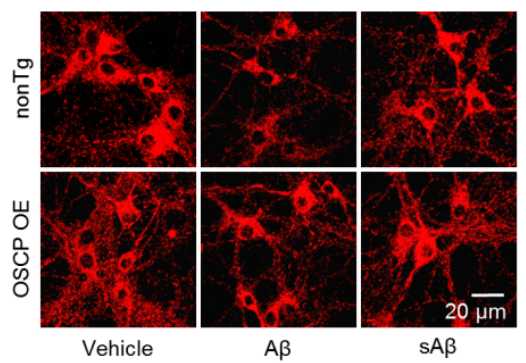

C2

B

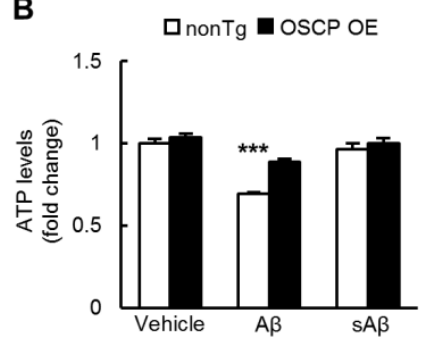

Mitosox Red Mitotracker Green

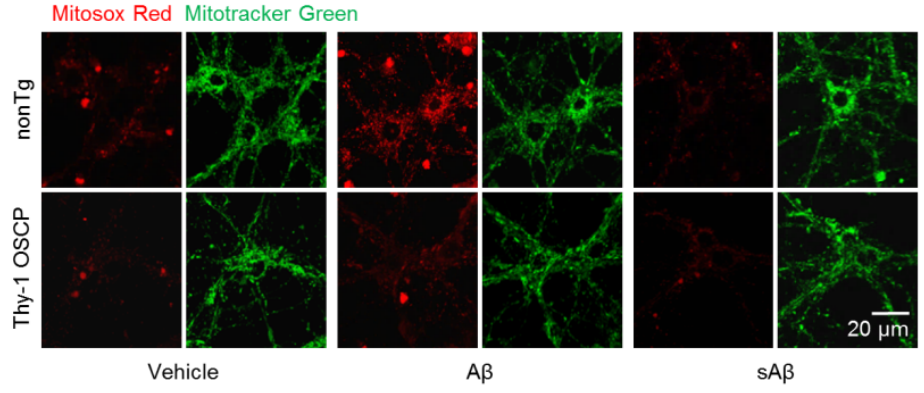

D3

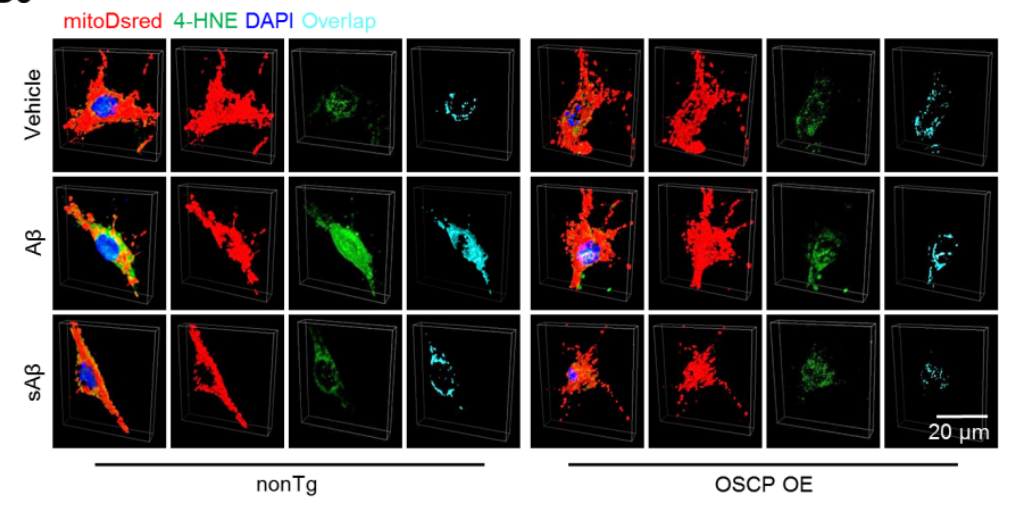

sFig. 4 Protected mitochondrial function in A $\beta$-treated OSCP overexpressing neurons. (AD) Primary nonTg and Thy1-OSCP neurons were treated with vehicle, $1 \mu \mathrm{M} A \beta 1-42$ or $1 \mu \mathrm{M}$ scramble $A \beta$ for 24 hours. (A1) Mitochondrial membrane potential was examined by TMRM staining. (A2) Representative images of TMRM stained mitochondria in primary neurons. Twoway ANOVA followed by Bonferroni post hoc analysis. *** $P<0.001$. Scale bar $=20 \mu \mathrm{m} . n=$ 26-32 neurons. (B) ATP level from cultured neurons. Data were collected from 4 independent experiments. (C1) Mitochondrial superoxide generation was examined by Mitosox staining. Mitotracker Green represents mitochondria. Two-way ANOVA followed by Bonferroni post hoc analysis. ${ }^{* * *} P<0.001 n=53-73$ neurons. (C2) Representative images of Mitosox Red (red color) staining in primary neurons. Mitochondria were labeled with Mitotracker green (green color). Scale bar $=20 \mu \mathrm{m}$. (D1\&D2) Lipid oxidation was examined by 4-HNE staining in neurons (D1) or mitochondria (D2). Two-way ANOVA followed by Bonferroni post hoc 
analysis. ${ }^{* *} P<0.01 . n=6-12$ neurons. (D3) Representative 3D images of 4-HNE staining (green color) in primary neurons. MitoDsred represents mitochondria. The overlaid staining of mitoDsred and 4-HNE represents Lipid oxidation in mitochondria. Scale bar $=10 \mu \mathrm{m}$. 
A

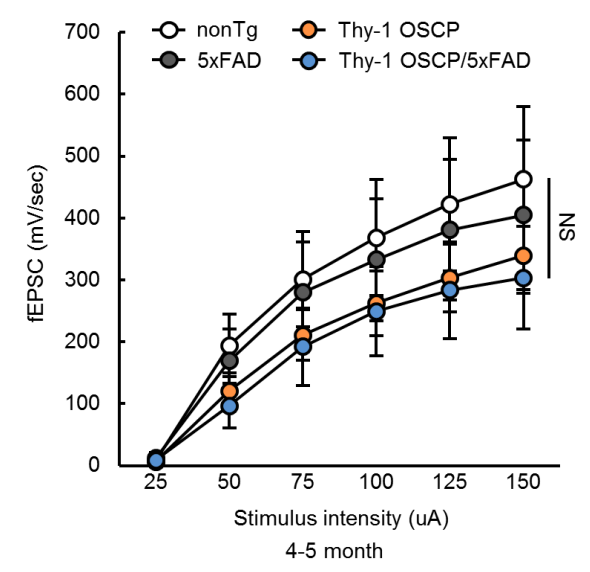

B

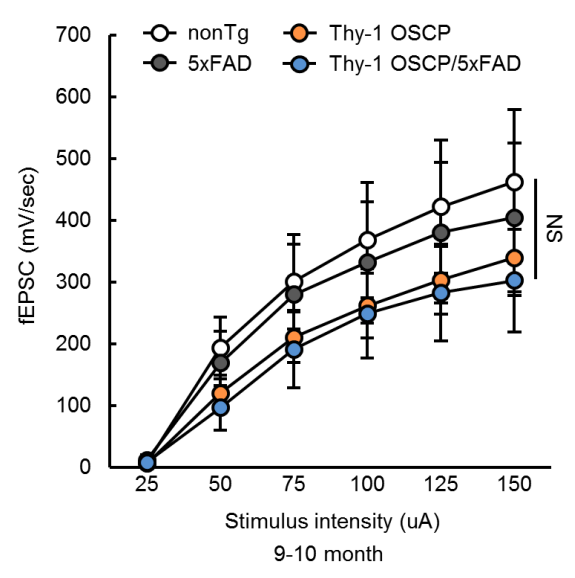

sFig. 5 Input/output curves of fEPSPs were similar in four types of transgenic mice at both ages. (A\&B) Input/output (I/O) curves of fEPSPs were obtained by plotting the slope of fEPSPs recorded in the CA1 area of the hippocampus as a function of the stimulation intensity (from 25 to $150 \mu \mathrm{A}$ ). Two-way ANOVA followed by Bonferroni post hoc analysis. NS, not significant. 45-month nonTg, $n=6$; 5xFAD, $n=8$; Thy-1 OSCP, $n=7$, Thy-1 OSCP/5xFAD, $n=8$; 9-10month nonTg, $n=8$; 5xFAD, $n=9$; Thy-1 OSCP, $n=8$, Thy-1 OSCP $/ 5 x F A D, n=9$. 

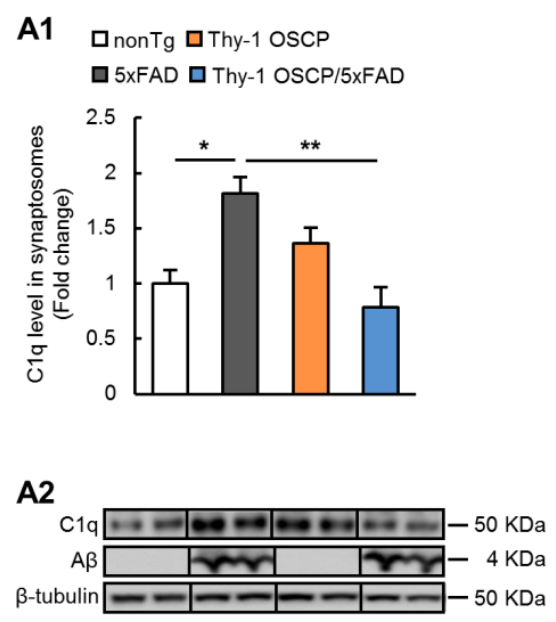

sFig. 6 Increased C1q level in isolated synaptosome. Immunoblotting analysis of C1q in isolated synaptosome from 9-10-month-old nonTg, 5xFAD, Thy-1 OSCP, and Thy-1 OSCP/5xFAD mice. Two-way ANOVA followed by Bonferroni post hoc analysis. ${ }^{*} P<0.05$, ** $P<0.01 . n=3$ each group. (B2) Representative bands of immunoblotting of $\mathrm{C} 1 \mathrm{q}, \mathrm{A} \beta$ and $\beta$ actin. $\beta$-actin was used as a loading control. 